\title{
Lifting at higher levels in the D1D5 CFT
}

\section{Bin Guo and Samir D. Mathur}

Department of Physics, The Ohio State University, Columbus, OH 43210, U.S.A.

E-mail: guo.1281@osu.edu, mathur.16@osu.edu

ABSTRACT: The D1D5P system has a large set of BPS states at its orbifold point. Perturbing away from this 'free' point leads to some states joining up into long supermultiplets and lifting, while other states remain BPS. We consider the simplest orbifold which exhibits this lift: that with $N=2$ copies of the free $c=6$ CFT. We write down the number of lifted and unlifted states implied by the index at all levels upto 6 . We work to second order in the perturbation strength $\lambda$. For levels upto 4, we find the wavefunctions of the lifted states, their supermultiplet structure and the value of the lift. All states that are allowed to lift by the index are in fact lifted at order $O\left(\lambda^{2}\right)$. We observe that the unlifted states in the untwisted sector have an antisymmetry between the copies in the right moving Ramond ground state sector, and extend this observation to find classes of states for arbitrary $N$ that will remain unlifted to $O\left(\lambda^{2}\right)$.

Keywords: Conformal Field Theory, Extended Supersymmetry, AdS-CFT Correspondence, Black Holes in String Theory

ARXIV EPRINT: 2008.01274 


\section{Contents}

1 Introduction $\quad 2$

2 The D1D5 CFT 3

2.1 Symmetries of the CFT 4

2.2 Deformation of the CFT 4

3 Computation of the lift using the Gava-Narain method 5

4 The character decomposition $\quad 7$

4.1 The partition function 7

4.2 The character decomposition 8

4.3 The constraints on lifting from the index 9

5 The method for finding the lifted primaries $\quad 10$

$\begin{array}{lll}5.1 & \text { Right movers } & 10\end{array}$

$\begin{array}{ll}5.2 \text { Left movers } & 11\end{array}$

6 Long multiplets and lifting $\quad 12$

$\begin{array}{lll}6.1 & \text { Level-1 } & 12\end{array}$

$\begin{array}{lll}6.2 & \text { Level-2 } & 13\end{array}$

$\begin{array}{lll}6.3 & \text { Level-3 } & 14\end{array}$

$\begin{array}{lll}6.4 & \text { Level-4 } & 15\end{array}$

6.5 Properties of lifting at $O\left(\lambda^{2}\right) \quad 16$

7 Unlifted states in the untwisted sector $\quad 16$

$\begin{array}{lll}7.1 & \text { Right movers for the }(1,1) \text { sector } & 16\end{array}$

$\begin{array}{lll}7.2 & \text { More general states } & 17\end{array}$

$\begin{array}{lll}7.3 & \text { Strings with winding numbers } k_{1} \text { and } k_{2} & 18\end{array}$

8 Discussion 19

A Contracted large $\mathcal{N}=4$ superconformal algebra $\quad 20$

B The $\mathcal{N}=4$ character $\quad 22$

B.1 The small $\mathcal{N}=4$ character 22

B.2 The contracted large $\mathcal{N}=4$ character 22

C The effect of the twist operator $\quad 23$

D Twisting strings with winding numbers $k_{1}$ and $k_{2} \quad 24$ 


\section{Introduction}

Black holes in string theory must be made by taking bound states of objects - strings and branes - present in the theory. The D1D5P system provides a very useful example of such a construction. One finds that the entropy and the rate of low energy emission from string states matches the expectations from gravitational thermodynamics [1-4]. The D1 and D5 branes form a bound state whose dynamics can be given as an effective $1+1$ dimensional conformal field theory. The momentum charge $\mathrm{P}$ is given by the difference in energy between the left moving and right moving excitations in this CFT.

The CFT has a 'free point' which is given by a $1+1$ dimensional sigma model whose target space is an orbifold [5-12]. The orbifold theory consists of $N$ copes of a $c=6$ CFT, joined up in different 'twist sectors'. In each twist sector the excitations are just given by free left and right moving bosons and fermions, with an overall symmetry condition to enforce the orbifold symmetry. At this orbifold point, any state with no right moving oscillator excitations is extremal.

The situation changes as we deform the theory away from the orbifold point. Sets of extremal states can join up into larger multiplets and lift to higher energies, leaving a smaller set of states that remain extremal. The count of states that remain unlifted is given by an index. This index was computed in [1] for the case where the compactification is $K 3 \times S^{1}$ and in [13] for the compactification $T^{4} \times S^{1}$. Our interest is in finding the actual states that are unlifted, the supermultiplet structure for groups of states that do lift, and the value of this lift. In particular we are interested in understanding the value of the twist for sectors where typical lifted and unlifted states arise, since this is relevant for the physical picture of the extremal hole. States in highly twisted sectors correspond to gravity states with deep throats, while states in sectors with low twist describe shallow throats. (For constructions of 'fuzzball' microstates dual to different CFT states see for example [14-18].)

In [19] the lift was computed, in a certain approximation scheme, for the situation where most of the CFT copies are in the untwisted sector and one set is in a twisted sector. Low energy excitations of this sector can be mapped, in the gravity dual, to strings in an $A d S_{3} \times S^{3} \times T^{4}$ spacetime. Apart from a small set of states in the graviton multiplet, these string states are all lifted. On the other hand we know from the index computation of [13] that if we go to sufficiently high energies and twists to reach black hole states, then a large number of states must remain unlifted: the index of [13] agrees with the Bekenstein entropy of extremal holes for large charges. It would be very interesting to understand better what properties of the highly excited states makes them remain 'unlifted'.

In this paper, we consider a CFT with $N=2$; this means that the product of the number $N_{1}$ of D1 branes and $N_{5}$ of D5 branes is $N_{1} N_{5} \equiv N=2$. We work to second order in the perturbation off the orbifold point. In [20] this problem was studied for the lowest nontrivial amount of momentum charge $P=1$, and the pattern of lifting was found. In the present paper, we will extend the results to $P=4$ and make some observations about the general nature of the unlifted states. 
Our steps and results are as follows:

(i) We study the constraints on lifting from the index. We find the number of states that can be lifted upto level $P=6$. We describe the structure of the long supermultiplets which relate these states. We identify the class of states that can be lowest members of long supermultiplets.

(ii) We give explicitly the wavefunctions for these lowest members of the long supermultiplets, upto level $P=3$. (The wavefunctions for $P=4$ is found in a similar way but the expressions are rather unwieldy, so we do not write them down in this paper.) We calculate the lift for all states to level $P=4$ at $O\left(\lambda^{2}\right)$ in the perturbation. We find that at this order the lift is nonzero for all these states. Thus all states upto this level that are allowed to lift by the index are in fact lifted at order $O\left(\lambda^{2}\right)$.

(iii) We discuss the general nature of states that remain unlifted at $O\left(\lambda^{2}\right)$. First consider the case $N=2$, and states in the untwisted sector. Let the left and right sectors both be fermionic, so that the overall state of each multiwound copy is bosonic. We observe that if the right moving sector is antisymmetric in the two copies, then the state will remain unlifted at $O\left(\lambda^{2}\right)$. We then extend this observation to the case $N>2$, including the situation where the component strings that are joined may have windings $k_{1}, k_{2}$ greater than unity.

Before proceeding, we note that there are many earlier works that study conformal perturbation theory, the lifting of the states, the acquiring of anomalous dimensions, and the issue of operator mixing, in particular in the context of the D1D5 CFT see for example [21-35]. Also, for more computations in conformal perturbation theory in two and higher dimensional CFTs see, e.g. [36-47].

\section{The D1D5 CFT}

In this section, we summarize some properties of the D1D5 CFT at the orbifold point and the deformation operator that we will use to perturb away from the orbifold point. For more details, see [21, 22].

Consider type IIB string theory, compactified as

$$
M_{9,1} \rightarrow M_{4,1} \times S^{1} \times T^{4}
$$

Wrap $N_{1}$ D1 branes on $S^{1}$, and $N_{5}$ D5 branes on $S^{1} \times T^{4}$. The bound state of these branes is described by a field theory. We think of the $S^{1}$ as being large compared to the $T^{4}$, so that at low energies we look for excitations only in the direction $S^{1}$. This low energy limit gives a conformal field theory (CFT) on the circle $S^{1}$.

It has been conjectured that we can move in the moduli space of couplings in the string theory to a point called the 'orbifold point' where the CFT is particularly simple. At this orbifold point the CFT is a $1+1$ dimensional sigma model. We will work in the Euclidized theory, where the base space is a cylinder spanned by the coordinates

$$
\tau, \sigma: \quad 0 \leq \sigma<2 \pi, \quad-\infty<\tau<\infty
$$


The target space of the sigma model is the 'symmetrized product' of $N_{1} N_{5}$ copies of $T^{4}$,

$$
\left(T^{4}\right)^{N_{1} N_{5}} / S_{N_{1} N_{5}}
$$

with each copy of $T^{4}$ giving 4 bosonic excitations $X^{1}, X^{2}, X^{3}, X^{4}$. It also gives 4 fermionic excitations, which we call $\psi^{1}, \psi^{2}, \psi^{3}, \psi^{4}$ for the left movers, and $\bar{\psi}^{1}, \bar{\psi}^{2}, \bar{\psi}^{3}, \bar{\psi}^{4}$ for the right movers. The fermions can be antiperiodic or periodic around the $\sigma$ circle. If they are antiperiodic on the $S^{1}$ we are in the Neveu-Schwarz (NS) sector, and if they are periodic on the $S^{1}$ we are in the Ramond (R) sector. The central charge of the theory with fields $X^{i}, \psi^{i}, i=1 \ldots 4$ is $c=6$. The total central charge of the entire system is thus

$$
c=6 N_{1} N_{5} \equiv 6 N
$$

\subsection{Symmetries of the CFT}

The D1D5 CFT has $(4,4)$ supersymmetry, which means that we have $\mathcal{N}=4$ supersymmetry in both the left and right moving sectors. This leads to a superconformal $\mathcal{N}=4$ symmetry in both the left and right sectors, generated by operators $L_{n}, G_{ \pm, r}^{ \pm}, J_{n}^{a}$ for the left movers and $\bar{L}_{n}, \bar{G}_{ \pm, r}^{ \pm}, \bar{J}_{n}^{a}$ for the right movers. The full symmetry is actually larger: it is the contracted large $\mathcal{N}=4$ superconformal symmetry [13, 48]. The algebra generators and commutators are given in appendix A.

Each $\mathcal{N}=4$ algebra has an internal $\mathrm{R}$ symmetry group $\mathrm{SU}(2)$, so there is a global symmetry group $\mathrm{SU}(2)_{L} \times \mathrm{SU}(2)_{R}$. We denote the quantum numbers in these two $\mathrm{SU}(2)$ groups as

$$
\mathrm{SU}(2)_{L}:(j, m) ; \quad \mathrm{SU}(2)_{R}:(\bar{j}, \bar{m})
$$

In the geometrical setting of the CFT, this symmetry arises from the rotational symmetry in the 4 space directions of $M_{4,1}$ : we have $\mathrm{SO}(4)_{E} \simeq \mathrm{SU}(2)_{L} \times \mathrm{SU}(2)_{R}$. Here the subscript $E$ stands for 'external', which denotes that these rotations are in the noncompact directions. We have another $\mathrm{SO}(4)$ symmetry in the four directions of the $T^{4}$. This symmetry we call $\mathrm{SO}(4)_{I}$ (where $I$ stands for 'internal'). This symmetry is broken by the compactification of the torus, but at the orbifold point it still provides a useful organizing principle. We write $\mathrm{SO}(4)_{I} \simeq \mathrm{SU}(2)_{1} \times \mathrm{SU}(2)_{2}$. We use spinor indices $\alpha, \bar{\alpha}$ for $\mathrm{SU}(2)_{L}$ and $\mathrm{SU}(2)_{R}$ respectively. We use spinor indices $A, \dot{A}$ for $\mathrm{SU}(2)_{1}$ and $\mathrm{SU}(2)_{2}$ respectively.

The 4 real fermions of the left sector can be grouped into complex fermions $\psi^{\alpha A}$. The right fermions have indices $\bar{\psi}^{\bar{\alpha} A}$. The bosons $X^{i}$ are a vector in the $T^{4}$. One can decompose this vector into the $\left(\frac{1}{2}, \frac{1}{2}\right)$ representation of $\mathrm{SU}(2)_{1} \times \mathrm{SU}(2)_{2}$, which gives scalars $X_{A \dot{A}}$.

\subsection{Deformation of the CFT}

The deformation of the CFT off the orbifold point is given by adding a deformation operator $D$ to the Lagrangian

$$
S \rightarrow S+\lambda \int d^{2} z D(z, \bar{z})
$$

where $D$ has conformal dimensions $(h, \bar{h})=(1,1)$. A choice of $D$ which is a singlet under all the symmetries at the orbifold point is

$$
D=\frac{1}{4} \epsilon^{\dot{A} \dot{B}} \epsilon_{\alpha \beta} \epsilon_{\bar{\alpha} \bar{\beta}} G_{\dot{A},-\frac{1}{2}}^{\alpha} \bar{G}_{\dot{B},-\frac{1}{2}}^{\bar{\alpha}} \sigma^{\beta \bar{\beta}}
$$


where $\sigma^{\beta \bar{\beta}}$ is a twist operator of rank 2 in the orbifold theory. Here $G$ and $\bar{G}$ are the left and right moving supercharge operators at the orbifold point.

\section{Computation of the lift using the Gava-Narain method}

We are interested in finding states which have well defined scaling dimensions, and the values of these dimensions, as we move away from the orbifold point. We will work in the Ramond sector. We measure the dimensions from the Ramond ground state, which is $\left(\frac{c}{24}, \frac{c}{24}\right)$. WE use the term 'level $n$ ' for the states with dimensions

$$
(h, \bar{h})=(n, 0)
$$

Let these states be labelled by indices $a, b, \ldots$, and written as $\left|O_{a}^{(0)}\right\rangle$ etc.

It turns out that while such states receive corrections at first order in $\lambda$, the dimensions get corrections only starting at $O\left(\lambda^{2}\right)$. The computation involves pulling down two copies of the deformation operator $D$ from the action, and then integrating the positions of these two $D$ operators. We first compute the matrix elements

$$
X_{b a}(T)=\left\langle O_{b}^{(0)}\left(\frac{T}{2}\right)\left|\left(\int d^{2} w_{1} D\left(w_{1}, \bar{w}_{1}\right)\right)\left(\int d^{2} w_{2} D\left(w_{2}, \bar{w}_{2}\right)\right)\right| O_{a}^{(0)}\left(-\frac{T}{2}\right)\right\rangle
$$

Then we compute the matrix

$$
E_{b a}^{(2)}=\lim _{T \rightarrow \infty}-\frac{\lambda^{2}}{2 T} e^{E^{(0)} T} X_{b a}(T)
$$

where $E^{(0)}$ is the energy of the states $\left|O_{a}^{(0)}\right\rangle$ at the orbifold point. The eigenstates of this matrix then give the linear combinations of the $\left|O_{a}^{(0)}\right\rangle$ which have definite dimensions and the eigenvalues give the lift in energy of the corresponding states.

Such $O\left(\lambda^{2}\right)$ corrections were computed for some simple states in $[44,45]$. In general the computation of a correlation functions with deformation operators involves going to a covering space where the effect of the twists is undone, and one gets a correlator of operators not involving twists on this covering space. But the covering space can be a sphere in some cases, and a torus in other cases. While correlators on a sphere are easy to compute, they can be difficult to find on a torus. (A central reason for this difficulty is that the correlators on the covering space can involve spin fields. On a sphere we can remove these spin fields by spectral flows, but it is not clear how to do this on a higher genus surface.)

If we cannot explicitly compute the amplitudes (3.2), then how can we find the lifting? In [19] Gava and Narain gave a method by which amplitudes like (3.2) could be written as modulus squared of amplitudes involving just one twist. Computing these one-twist amplitudes always gives a covering space that is a sphere, so the computation is straightforward.

In [49] this proposal of [19] was studied in detail. Let us recall the results of this study. We find

$$
\epsilon_{\dot{A} \dot{B}} \epsilon^{\bar{\alpha} \bar{\beta}} E_{b a}^{(2)}=2 \lambda^{2}\left\langle O_{b}^{(0)}\left|\left\{\bar{G}_{\dot{A}, 0}^{\bar{\alpha}(P)}, \bar{G}_{\dot{B}, 0}^{\bar{\beta}(P)}\right\}\right| O_{a}^{(0)}\right\rangle
$$


We will refer to the matrix $E_{b a}^{(2)}$ as the lifting matrix $E^{(2)}$. The operators $\bar{G}_{\dot{A}, 0}^{\bar{\alpha}(P)}$ are defined as

$$
\bar{G}_{\dot{A}, 0}^{\bar{\alpha}(P)}=\pi \mathcal{P} G_{\dot{A},-\frac{1}{2}}^{+} \sigma^{-\bar{\alpha}} \mathcal{P}
$$

where the operator $\mathcal{P}$ is a projection operator, which projects any state to the subspace spanned by the unperturbed states $\left|O_{a}^{(0)}\right\rangle$ which have the dimensions (3.1).

From the above relation we see that the lifting matrix (3.3) can be written using either of the following two equivalent expressions

$$
E_{b a}^{(2)}=2 \lambda^{2}\left\langle O_{b}^{(0)}\left|\left\{\bar{G}_{+, 0}^{+(P) \dagger}, \bar{G}_{+, 0}^{+(P)}\right\}\right| O_{a}^{(0)}\right\rangle=2 \lambda^{2}\left\langle O_{b}^{(0)}\left|\left\{\bar{G}_{-, 0}^{+(P) \dagger}, \bar{G}_{-, 0}^{+(P)}\right\}\right| O_{a}^{(0)}\right\rangle
$$

Further, it was noted in [49] that the operators $\bar{G}_{\dot{A}, 0}^{\bar{\alpha}(P)}$ give the supersymmetric structure of long multiplets. At the orbifold point the states can be grouped into short multiplets. As we deform away from the orbifold point, four of these short multiplets can join into a long multiplet and lift. The structure of this long multiplet is indicated in the following diagram:
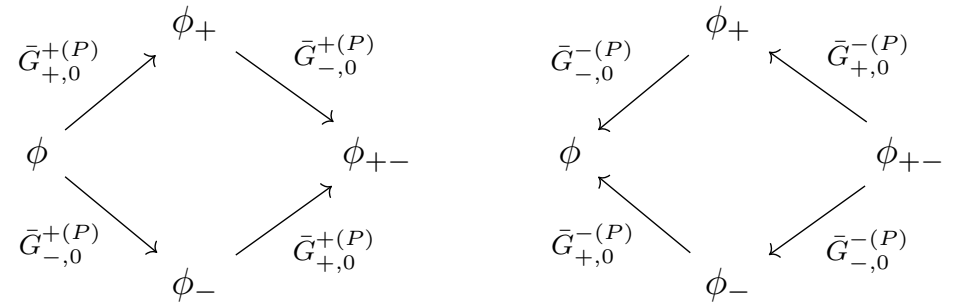

The state $\phi$ is at the bottom of this long multiplet. Note that $\phi$ is a member of a short multiplet created by operators that are not depicted in the diagram. The operators $\bar{G}_{+, 0}^{+(P)}$ and $\bar{G}_{-, 0}^{+(P)}$ play the role of the two raising operators which take us to states $\phi_{+}, \phi_{-}$which are members of two other short multiplets. Acting with both these raising operators takes us to the short multiplet represented by the state $\phi_{+-}$. We can move along this multiplet in the reverse direction using the lowering operators $\bar{G}_{+, 0}^{-(P)}$ and $\bar{G}_{-, 0}^{-(P)}$.

Suppose we have diagonalized the matrix $E^{(2)}$ given in eq. (3.6). Let $\left|O^{(0)}\right\rangle$ be an eigenstate of this matrix. Let the corresponding eigenvalue, which gives the lift of this operator, be called $E_{O}^{(2)}$. Then from (3.6) we find that $E_{O}^{(2)}$ can be written as a sum of modulus-squared terms

$$
\left.\left.\left.\left.E_{O}^{(2)}=\left.2 \lambda^{2}\left(\left|\bar{G}_{+, 0}^{+(P)}\right| O^{(0)}\right\rangle\right|^{2}+\left|\bar{G}_{-, 0}^{-(P)}\right| O^{(0)}\right\rangle\left.\right|^{2}\right)=\left.2 \lambda^{2}\left(\left|\bar{G}_{-, 0}^{+(P)}\right| O^{(0)}\right\rangle\right|^{2}+\left|\bar{G}_{+, 0}^{-(P)}\right| O^{(0)}\right\rangle\left.\right|^{2}\right)
$$

In the long multiplet described in (3.7) each of the four states $\phi, \phi_{+}, \phi_{-}$and $\phi_{+-}$ have the following property: if it can be raised by $\bar{G}_{+, 0}^{+(P)}$, then it will be annihilated by the $\bar{G}_{-, 0}^{-(P)}$; conversely, if it can be lowered by $\bar{G}_{-, 0}^{-(P)}$ then it will be annihilated by the $\bar{G}_{+, 0}^{+(P)}$. A similar statement holds for the raising operators $\bar{G}_{-, 0}^{+(P)}$ and the lowering operators $\bar{G}_{+, 0}^{-(P)}$. Thus in each of the two expressions in (3.8), only one of the two terms is nonzero.

In summary, one can get the value of the lift and the corresponding eigenstates by diagonalizing the lifting matrix $E^{(2)}(3.6)$. Four short multiplets join into a long multiplet as shown in eq. (3.7) and the lifting can be calculated from (3.8). 
In the cases that we will encounter below, there is a unique state $\left|O_{a}^{(0)}\right\rangle$ with the relevant quantum numbers, so we will not have to diagonalize a matrix $E_{b a}^{(2)}$ to first find the eigenvectors $\left|O^{(0)}\right\rangle$.

\section{The character decomposition}

The CFT has a left and a right moving superconformal symmetry, with each of these described by the contracted large $\mathcal{N}=4$ superconformal algebra [13, 48]. The algebra generators and commutation relations are given in appendix $\mathrm{A}$.

These symmetries remain true for all values of the coupling. Thus states related by these symmetries will have the same lift $E^{(2)}$. We would like to group states into multiplets that are related by these symmetries, so that we can reduce the number of independent lifting computations that we have to perform. In this section, we will count the number of multiplets by using the character decompostion (see the related works $[50,51]$ ). The main result of this section is given in table 1 below.

In subsection 4.1, we recall the partition function of the orbifold theory. In subsection 4.2 , we count the number of multiplets by writing the partition function in terms of characters. In subsection 4.3, we consider the constraints on lifting that arise from considering the index.

\subsection{The partition function}

In this subsection we recall the partition function of the orbifold CFT. The partition function for a single $c=6$ copy of the CFT is defined as

$$
Z=\operatorname{Tr}(-1)^{2 J_{0}^{3}-2 \bar{J}_{0}^{3}} q^{L_{0}-c / 24} \bar{q}^{\bar{L}_{0}-c / 24} y^{2 J_{0}^{3}} \bar{y}^{2 \bar{J}_{0}^{3}} \equiv \sum_{h, \bar{h}, j_{3}, \bar{j}_{3}} c\left(h, \bar{h}, j_{3}, \bar{j}_{3}\right) q^{h} \bar{q}^{\bar{h}} y^{2 j_{3}} \bar{y}^{2 \bar{j}_{3}}
$$

We work with the case where the 4-manifold is $T^{4}$. There are $\mathrm{U}(1)$ charges that arise from momentum and winding charges around this $T^{4}$, but we work in the sector where all such charges have been taken to be zero. For this choice, one finds

$$
Z\left(T^{4}\right)=\left(\frac{\theta_{1}}{\eta}\right)^{2} \frac{1}{\eta^{4}} \overline{\left(\frac{\theta_{1}}{\eta}\right)^{2} \frac{1}{\eta^{4}}}
$$

where

$$
\begin{aligned}
\theta_{1} & =i\left(y^{1 / 2}-y^{-1 / 2}\right) q^{1 / 8} \prod_{n=1}^{\infty}\left(1-q^{n}\right)\left(1-y q^{n}\right)\left(1-y^{-1} q^{n}\right) \\
\eta & =q^{1 / 24} \prod_{n=1}^{\infty}\left(1-q^{n}\right)
\end{aligned}
$$

Using the above we can find the partition function for the case where the target space of the $1+1$ dimensional CFT is the symmetric product $\operatorname{Sym}^{N}\left(T^{4}\right)$. The partition function for a symmetric product target space $Z\left(\operatorname{Sym}^{N}(X)\right)$ is given by

$$
\mathcal{Z}(p, q, \bar{q}, y, \bar{y})=\sum_{N=0}^{\infty} p^{N} Z\left(\operatorname{Sym}^{N}(X)\right)=\prod_{n=1}^{\infty} \prod_{h, \bar{h}, j_{3}, \bar{j}_{3}}^{\prime} \frac{1}{\left(1-p^{n} q^{h / n} \bar{q}^{\bar{h} / n} y^{2 j_{3}} \bar{y}^{2 \bar{j}_{3}}\right)^{c\left(h, \bar{h}, j_{3}, \bar{j}_{3}\right)}}
$$


where $\prod_{h, \bar{h}, j_{3}, \bar{j}_{3}}^{\prime}$ is restricted so that $(h-\bar{h}) / n$ is an integer. The $c\left(h, \bar{h}, j_{3}, \bar{j}_{3}\right)$ are the degeneracies appearing in the partition function for the CFT with a single copy of the space $X$. We write

$$
\mathcal{Z}(p, q, \bar{q}, y, \bar{y})=\sum_{N, h, \bar{h}, j_{3}, \bar{j}_{3}} c\left(N, h, \bar{h}, j_{3}, \bar{j}_{3}\right) p^{N} q^{h} \bar{q}^{\bar{h}} y^{2 j_{3}} \bar{y}^{2 \overline{j_{3}}}
$$

This yields the degeneracies $c\left(N, h, \bar{h}, j_{3}, \bar{j}_{3}\right)$ of the states with total winding of the effective string $N$ and quantum number $h, \bar{h}, j_{3}, \bar{j}_{3}$.

\subsection{The character decomposition}

We now write the partition function in terms of the characters of the contracted large $\mathcal{N}=4$ superconformal symmetry. We let the total winding number of the effective string be $N=2$, and consider states with $\bar{h}=0$. From (4.4) and (4.5), the partition function for this case is

$$
Z(N=2 ; \bar{h}=0)=\sum_{h, j_{3}, \bar{j}_{3}} c\left(N=2, h, \bar{h}=0, j_{3}, \bar{j}_{3}\right) q^{h} y^{2 j_{3}} \bar{y}^{2 \bar{j}_{3}}
$$

There are two different twist sectors for $N=2$ : the case of two singly wound copies which we call $N=(1,1)$, and the case of a single doubly wound copy which we call $N=(2)$. The expression (4.6) includes the contribution from both these twist sectors. We are however interested in obtaining the contribution separately from these two different twist sectors. It turns out that with a little effort we can separate the two contributions in the expression (4.6). This is done as follows:

$N=(1,1)$ sector: in $(4.4)$, restrict the product to terms with $n=1$ and collect all the terms with dependence $p^{2}$.

$N=(2)$ sector: in (4.4), restrict the product to terms with $n=2$ and collect all the terms with dependence $p^{2}$.

In this way, we can separate the two contributions

$$
Z(N=2 ; \bar{h}=0)=Z(N=(1,1) ; \bar{h}=0)+Z(N=(2) ; \bar{h}=0)
$$

For each of these two sectors, we find the following character decomposition

$$
Z(q, y, \bar{y})=\sum_{\substack{j=1 / 2,1 \\ \bar{j}_{3}=-1,-1 / 2,0}} c_{j ; \bar{j}_{3}}^{s} \chi_{j}^{s}(q, y) \bar{\chi}_{\bar{j}_{3}}(\bar{y})+\sum_{\substack{j=1 \\ \bar{j}_{3}=-1,-1 / 2,0}} c_{j, h ; \bar{j}_{3}}^{l} \chi_{j, h}^{l}(q, y) \bar{\chi}_{\bar{j}_{3}}(\bar{y})
$$

where $\chi_{j}^{s}$ and $\chi_{j, h}^{l}$ are given in appendix B. They are characters of a left moving contracted large $\mathcal{N}=4$ algebra. The $\chi_{j}^{s}$ is the character for a short representation in which the primary has dimension $h=0$. The $\chi_{j, h}^{l}$ is the character for a long representation in which the primary has dimension $h>0$. From appendix B for $N=2$, we see that the possible values of $j$ for a short representation are $j=1 / 2,1$ and for a long representation is $j=1$.

For the character $\bar{\chi}_{\bar{j}_{3}}$ of the right mover, we consider the sub-algebra formed by $\bar{d}_{0}^{\bar{\alpha} A}$. The lowest weight states are defined by

$$
\bar{d}_{0}^{-A}|\phi\rangle=0
$$




\begin{tabular}{|c|c|c|c|c|c|c|}
\hline level & sector & $\chi_{j=1, h}^{l} \bar{\chi}_{\bar{j}_{3}=-1}$ & $\chi_{j=1, h}^{l} \bar{\chi}_{\bar{j}_{3}=-1 / 2}$ & $\chi_{j=1, h}^{l} \bar{\chi}_{\bar{j}_{3}=0}$ & sector & $\chi_{j=1, h}^{l} \bar{\chi}_{\bar{j}_{3}=-1 / 2}$ \\
\hline & & $\phi$ & unlifted & $\phi_{+-}$ & & $\phi_{+}, \phi_{-}$, unlifted \\
\hline$h=1$ & $(1,1)$ & 3 & 0 & 3 & $(2)$ & 6 \\
\hline$h=2$ & $(1,1)$ & 1 & 16 & 1 & $(2)$ & 28 \\
\hline$h=3$ & $(1,1)$ & 18 & 8 & 18 & $(2)$ & 98 \\
\hline$h=4$ & $(1,1)$ & 15 & 72 & 15 & $(2)$ & 282 \\
\hline$h=5$ & $(1,1)$ & 68 & 80 & 68 & $(2)$ & 728 \\
\hline$h=6$ & $(1,1)$ & 89 & 264 & 89 & $(2)$ & 1734 \\
\hline
\end{tabular}

Table 1. The character decomposition at the orbifold point.

with charge

$$
\bar{J}_{0}^{3}|\phi\rangle=\bar{j}_{3}|\phi\rangle
$$

There are two fermionic raising operators $\bar{d}_{0}^{+ \pm}$. Application of these operators once to a lowest weight state, which has charge $\bar{j}_{3}$, gives two states with charges $\bar{j}_{3}+1 / 2$. Applying again gives a state with charge $\bar{j}_{3}+1$

$$
\bar{j}_{3} \rightarrow 2\left(\bar{j}_{3}+1 / 2\right) \rightarrow \bar{j}_{3}+1
$$

The character is defined by the trace over the irreducible representation. We find

$$
\bar{\chi}_{\bar{j}_{3}}(\bar{y})=\operatorname{Tr}(-1)^{-2 \bar{J}_{0}^{3}} \bar{y}^{2 \bar{J}_{0}^{3}}=-(-\bar{y})^{2 \bar{j}_{3}+1}\left(\bar{y}^{1 / 2}-\bar{y}^{-1 / 2}\right)^{2}
$$

The possible values of $\bar{j}_{3}$ for $N=2$ are $\bar{j}_{3}=-1,-1 / 2,0$. The results of the character decomposition (4.8) of $N=2$ are

(i) For the coefficients $c_{j ; \bar{j}_{3}}^{s}$ in (4.8), which tell us the numbers of primaries with dimension $h=0$ and with charges $j$ and $\bar{j}_{3}$, the results are

$$
\begin{aligned}
(1,1): & 4 \chi_{j=1 / 2}^{s} \bar{\chi}_{\bar{j}_{3}=-1 / 2}+\chi_{j=1}^{s} \bar{\chi}_{\bar{j}_{3}=-1}+\chi_{j=1}^{s} \bar{\chi}_{\bar{j}_{3}=0} \\
(2): & \chi_{j=1 / 2}^{s} \bar{\chi}_{\bar{j}_{3}=-1 / 2}
\end{aligned}
$$

(ii) For the coefficients $c_{j, h ; \bar{j}_{3}}^{l}$, which tell us the numbers of primaries with dimension $h>0$ and with charges $j$ and $\bar{j}_{3}$, the results are shown in table 1 .

\subsection{The constraints on lifting from the index}

Let us first recall how the index is computed. Consider the exact supercharge operators $\bar{G}_{\dot{A}}^{+}, \dot{A}=+,-$ of the perturbed CFT. These operators are the ones that join four short multiplets into a long multiplet. Let us see the structure of the set of states that will join into a long multiplet. Each of the operators $\bar{G}_{\dot{A}}^{+}$increases the $\mathrm{SU}(2)_{R}$ charge by $1 / 2$ and does not change the $\mathrm{SU}(2)_{L}$ charge. Thus the four short multiplets joining into a long multiplet must have the same left moving character but their right moving characters will be as follows:

$$
\bar{\chi}_{\bar{j}_{3}} \quad 2 \bar{\chi}_{\bar{j}_{3}+1 / 2} \quad \bar{\chi}_{\bar{j}_{3}+1}
$$


Whenever we can group states in the manner indicated by such a set of characters, then we find a set of states that have the charges to join into a long multiplet. We therefore exclude such sets of states from the index. If there are states left over that cannot group into a set with characters (4.14), then we count those states in the index, since they cannot possibly join into a long multiplet and lift.

Now let us look at table 1 . In the row $h=1$, it is possible to join $3 \chi_{j=1, h}^{l} \bar{\chi}_{\bar{j}_{3}=-1}$ and $3 \chi_{j=1, h}^{l} \bar{\chi}_{\bar{j}_{3}=0}$ in the $(1,1)$ sector and $6 \chi_{j=1, h}^{l} \bar{\chi}_{\bar{j}_{3}=-1 / 2}$ in the (2) sector into 3 long multiplets. In second order perturbation theory, they indeed join into 3 long multiplets [20]. In the row $h=2$, it is possible to join $\chi_{j=1, h}^{l} \bar{\chi}_{\bar{j}_{3}=-1}$ and $\chi_{j=1, h}^{l} \bar{\chi}_{\bar{j}_{3}=0}$ in $(1,1)$ sector and $2 \chi_{j=1, h}^{l} \bar{\chi}_{\bar{j}_{3}=-1 / 2}$ into a long multiplet. The 2 of $\chi_{j=1, h}^{l} \bar{\chi}_{\bar{j}_{3}=-1 / 2}$ can come from the 16 in the $(1,1)$ sector or the 28 in the $(2)$ sector. In second order perturbation theory, it should come from the (2) sector because the operators (3.5) joining short multiplets into long multiplets change the twist sector.

In general, in second order perturbation theory for any level $h>0$, the operators (3.5) join $\chi_{j=1, h}^{l} \bar{\chi}_{\bar{j}_{3}=-1}$ and $\chi_{j=1, h}^{l} \bar{\chi}_{\bar{j}_{3}=0}$ in the $(1,1)$ sector and $2 \chi_{j=1, h}^{l} \bar{\chi}_{\bar{j}_{3}=-1 / 2}$ in the $(2)$ sector into a long multiplet. In table 1 , the number in the column $\chi_{j=1, h}^{l} \bar{\chi}_{\bar{j}_{3}=-1 / 2}$ in the (2) sector grows faster than twice of the number in the column $\chi_{j=1, h}^{l} \bar{\chi}_{\bar{j}_{3}=-1}$ or $\chi_{j=1, h}^{l} \bar{\chi}_{\bar{j}_{3}=0}$ in the $(1,1)$ sector. Thus it is possible that all the states in the columns $\chi_{j=1, h}^{l} \bar{\chi}_{\bar{j}_{3}=-1}$ and $\chi_{j=1, h}^{l} \bar{\chi}_{\bar{j}_{3}=0}$ in the $(1,1)$ sector will be lifted by pairing with states in the (2) sector. In the following, we will find that states in these two columns with dimension up to $h=4$ are indeed lifted.

\section{The method for finding the lifted primaries}

The primaries and their descendents have same lift. Thus we can focus on the primaries, which are counted in table 1 . To find all the lifted primaries, we need to construct the primaries in the $\chi_{j=1, h}^{l} \bar{\chi}_{\bar{j}_{3}=-1}$ column explicitly. Then applying the two raising operators $\bar{G}_{ \pm, 0}^{+(P)}$ gives all the four lifted primaries in a long multiplet as in (3.7).

In subsection 5.1, we will construct the right movers of all the primaries explicitly. In subsection 5.2, we will construct the left movers of the primaries in the $\chi_{j=1, h}^{l} \bar{\chi}_{\bar{j}_{3}=-1}$ column up to level $h=4$.

\subsection{Right movers}

The right movers of primaries satisfying (4.9) with total winding $N=2$ were found in [20]. There are two classes:

(i) The following set of right moving states can join into a long multiplet and lift at second order in perturbation theory

$$
\begin{aligned}
(1,1) \quad \bar{\chi}_{\bar{j}_{3}=-1}: & \left|\phi^{R}\right\rangle=\left|\overline{0}_{R}^{-}\right\rangle\left|\overline{0}_{R}^{-}\right\rangle \\
(2) \quad \bar{\chi}_{\bar{j}_{3}=-1 / 2}: & \left|\phi_{+}^{R}\right\rangle=\left|\phi_{-}^{R}\right\rangle=\left|\overline{0}_{R}^{2-}\right\rangle \\
(1,1) \quad \bar{\chi}_{\bar{j}_{3}=0}: & \left|\phi_{+-}^{R}\right\rangle=\frac{1}{2}\left(\bar{d}_{0}^{++(1)}-\bar{d}_{0}^{++(2)}\right)\left(\bar{d}_{0}^{+-(1)}-\bar{d}_{0}^{+-(2)}\right)\left|\overline{0}_{R}^{-}\right\rangle\left|\overline{0}_{R}^{-}\right\rangle
\end{aligned}
$$


(ii) The following right moving state cannot join with other states into a long multiplet and thus states of this type cannot lift at second order in perturbation theory

$$
(1,1) \quad \bar{\chi}_{\bar{j}_{3}=-1 / 2}: \quad\left(\bar{d}_{0}^{+A(1)}-\bar{d}_{0}^{+A(2)}\right)\left|\overline{0}_{R}^{-}\right\rangle\left|\overline{0}_{R}^{-}\right\rangle
$$

The first and third right movers in (5.1) and the right mover in (5.2) are the three right moving primaries listed for the $(1,1)$ sector in table 1 . The second right mover in (5.1) is the right mover of the primary in the (2) sector in table 1.

The right mover of the operator $\bar{G}_{\dot{A}, 0}^{\bar{\alpha}(P)}$ is $\mathcal{P} \bar{\sigma}^{\bar{\alpha}}$. Thus the long multiplet structure (3.1) for the right movers is as given in the following diagram:
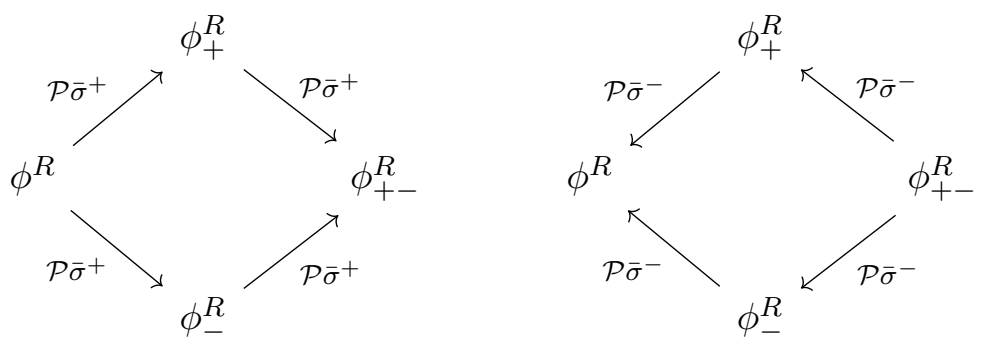

The states in class (i) satisfy the properties in (5.3). Thus they can join into a long multiplet and have nonzero lifting. The states in class (ii) are annihilated by $\mathcal{P} \bar{\sigma}^{\bar{\alpha}}$. Thus states who have right mover (5.2) have zero lift at $O\left(\lambda^{2}\right)$.

\section{$5.2 \quad$ Left movers}

In this subsection, we will explain how to find the left movers of primaries in the $\chi_{j=1, h}^{l} \bar{\chi}_{\bar{j}_{3}=-1}$ column in table 1.

In the $(1,1)$ sector, the global modes (A.10) are defined as

$$
O_{n}^{(g)}=O_{n}^{(1)}+O_{n}^{(2)}
$$

Thus these modes are applied symmetrically to the two copies of the CFT. The primaries $\phi$ with dimensions $h>0$ of the contracted large $\mathcal{N}=4$ superconformal algebra are defined by

$$
\begin{aligned}
L_{n}^{(g)}|\phi\rangle=G_{\dot{A}, n}^{+(g)}|\phi\rangle=J_{n}^{3(g)}|\phi\rangle & =J_{n}^{+(g)}|\phi\rangle=0 & & n>0 \\
G_{\dot{A}, n}^{-(g)}|\phi\rangle & =J_{n}^{-(g)}|\phi\rangle=0 & & n \geq 0
\end{aligned}
$$

and

$$
\alpha_{A \dot{A}, n}^{(g)}|\phi\rangle=d_{n}^{\alpha A(g)}|\phi\rangle=0 \quad n>0
$$

From the algebra in appendix A, all the conditions in eq. (5.5) follow from the conditions

$$
J_{0}^{-(g)}|\phi\rangle=J_{1}^{+(g)}|\phi\rangle=G_{\dot{A}, 0}^{-(g)}|\phi\rangle=0
$$

To find all states satisfying the relations (5.6), we define operators that are antisymmetric between the two copies:

$$
O_{n}^{(\mathcal{A})}=O_{n}^{(1)}-O_{n}^{(2)}
$$




\begin{tabular}{|c|c|c|c|}
\hline level & number of long multiplets & $\left(j_{A}, j_{\dot{A}}\right)$ & $E^{(2)} / \pi^{2} \lambda^{2}$ \\
\hline$h=1$ & 3 & $(1,0)$ & 1 \\
\hline$h=2$ & 1 & $(0,0)$ & $15 / 8$ \\
\hline$h=3$ & 18 & $(2,1),(1,0)$ & $3,64 / 39$ \\
\hline$h=4$ & 15 & $(1,1),(2,0),(0,0)$ & $3,5 / 2,2695 / 1024$ \\
\hline
\end{tabular}

Table 2. The energy lift at second order.

From the algebra in appendix A, one finds

$$
\left[\alpha_{A \dot{A}, n}^{(g)}, \alpha_{B \dot{B}, m}^{(\mathcal{A})}\right]=0, \quad\left\{d_{n}^{\alpha A(g)}, d_{m}^{\beta B(\mathcal{A})}\right\}=0
$$

Thus the states satisfying eq. (5.6) are the states built by acting with the antisymmetric operators $\alpha_{A \dot{A}, n}^{(\mathcal{A})}$ and $d_{n}^{\alpha A(\mathcal{A})}$ on the Ramond ground state $\left|0_{R}^{-}\right\rangle\left|0_{R}^{-}\right\rangle$.

Therefore, to find the primaries in the $\chi_{j=1, h}^{l} \bar{\chi}_{\bar{j}_{3}=-1}$ column, we can first build states using the antisymmetric operators $\alpha_{A \dot{A}, n}^{(\mathcal{A})}$ and $d_{n}^{\alpha A(\mathcal{A})}$. Then on this space of states we find the solutions of eq. (5.7). This procedure gives the required primaries. The results are described in section 6 below.

\section{$6 \quad$ Long multiplets and lifting}

In this section, we will find the long multiplets and their lift up to level-4. For each level, we will first construct the primaries in the $\chi_{j=1, h}^{l} \bar{\chi}_{\bar{j}_{3}=-1}$ column. Then we will apply the two raising operators $\bar{G}_{ \pm, 0}^{+(P)}$ to get all the four primaries in a long multiplet. Because the four primaries in a long multiplet have the same lift, we will only calculate the lift of the primaries in the $\chi_{j=1, h}^{l} \bar{\chi}_{\bar{j}_{3}=-1}$ column. For these states, the lift (3.8) becomes

$$
\left.\left.E_{\phi}^{(2)}=2 \lambda^{2}\left|\bar{G}_{+, 0}^{+(P)}\right| \phi\right\rangle\left.\right|^{2}=2 \lambda^{2}\left|\bar{G}_{-, 0}^{+(P)}\right| \phi\right\rangle\left.\right|^{2}
$$

In the following, we will organize primaries into $A$ and $\dot{A}$ charge multiplets, which will be labeled by $\left(j_{A}, j_{\dot{A}}\right)$. We will only write the result of the lowest weight states explicitly for each $A$ and $\dot{A}$ charge multiplet.

We find that up to level-4 all states that can lift do actually lift at second order. The lifts are listed in table 2.

\subsection{Level-1}

The 3 primaries in the $\chi_{j=1, h}^{l} \bar{\chi}_{\bar{j}_{3}=-1}$ column can be organized into a $A$ charge triplet and $\dot{A}$ charge singlet; we label this as $(1,0)$. 
For the lowest weight state of the $(1,0)$ multiplet, the normalized left movers of the long multiplet are

$$
\begin{aligned}
\left|\phi^{L}\right\rangle=\left|\phi_{+-}^{L}\right\rangle & =\frac{1}{2} d_{-1}^{--(\mathcal{A})} d_{0}^{+-(\mathcal{A})}\left|0_{R}^{-}\right\rangle\left|0_{R}^{-}\right\rangle \\
\left|\phi_{+}^{L}\right\rangle & =\frac{1}{\sqrt{2}} d_{-1 / 2}^{--} \alpha_{++,-1 / 2}\left|0_{R}^{2-}\right\rangle \\
\left|\phi_{-}^{L}\right\rangle & =\frac{1}{\sqrt{2}} d_{-1 / 2}^{--} \alpha_{+-,-1 / 2}\left|0_{R}^{2-}\right\rangle
\end{aligned}
$$

while the normalized right movers are in eq. (5.1). Applying (6.1) to the primary $\phi$, one finds the lift

$$
E_{1,(1,0)}^{(2)}=\pi^{2} \lambda^{2}
$$

\section{$6.2 \quad$ Level-2}

The one primary in the $\chi_{j=1, h}^{l} \bar{\chi}_{\bar{j}_{3}=-1}$ column is a $A$ charge singlet and $\dot{A}$ charge singlet; thus we have the representation $(0,0)$.

The normalized left movers of the long multiplet are

$$
\begin{aligned}
\left|\phi^{L}\right\rangle=\left|\phi_{+-}^{L}\right\rangle= & \frac{1}{2 \sqrt{10}}\left[d_{-1}^{-+(\mathcal{A})} d_{-1}^{--(\mathcal{A})} d_{0}^{+-(\mathcal{A})} d_{0}^{++(\mathcal{A})}\right. \\
& +d_{-1}^{-+(\mathcal{A})} d_{-1}^{+-(\mathcal{A})}+d_{-1}^{++(\mathcal{A})} d_{-1}^{--(\mathcal{A})}-d_{-2}^{-+(\mathcal{A})} d_{0}^{+-(\mathcal{A})}+d_{-2}^{-(\mathcal{A})} d_{0}^{++(\mathcal{A})} \\
& \left.-\alpha_{++,-1}^{(\mathcal{A})} \alpha_{--,-1}^{(\mathcal{A})}+\alpha_{-+,-1}^{(\mathcal{A})} \alpha_{+-,-1}^{(\mathcal{A})}\right]\left|0_{R}^{-}\right\rangle\left|0_{R}^{-}\right\rangle \\
\left|\phi_{+}^{L}\right\rangle= & \frac{1}{4 \sqrt{6}} \sum_{A=+,-}\left[\alpha_{A+,-3 / 2} d_{-1 / 2}^{-A}-3 \alpha_{A+,-1 / 2} d_{-3 / 2}^{-A}\right. \\
& -\frac{3}{2} \alpha_{A+,-1 / 2} d_{-1 / 2}^{+A} d_{-1 / 2}^{-+} d_{-1 / 2}^{--} \\
& \left.+\left(\alpha_{+-,-1 / 2} \alpha_{-+,-1 / 2}-\alpha_{++,-1 / 2} \alpha_{--,-1 / 2}\right) \alpha_{A+,-1 / 2} d_{-1 / 2}^{-A}\right]\left|0_{R}^{2-}\right\rangle \\
\left|\phi_{-}^{L}\right\rangle= & \frac{1}{4 \sqrt{6}} \sum_{A=+,-}\left[\alpha_{A-,-3 / 2} d_{-1 / 2}^{-A}-3 \alpha_{A-,-1 / 2} d_{-3 / 2}^{-A}\right. \\
& -\frac{3}{2} \alpha_{A-,-1 / 2} d_{-1 / 2}^{+A} d_{-1 / 2}^{-+} d_{-1 / 2}^{--} \\
& \left.+\left(\alpha_{+-,-1 / 2} \alpha_{-+,-1 / 2}-\alpha_{++,-1 / 2} \alpha_{--,-1 / 2}\right) \alpha_{A-,-1 / 2} d_{-1 / 2}^{-A}\right]\left|0_{R}^{2-}\right\rangle
\end{aligned}
$$

Applying (6.1) to the primary $\phi$, one finds the lift

$$
E_{2,(0,0)}^{(2)}=\frac{15}{8} \pi^{2} \lambda^{2}
$$




\subsection{Level-3}

The 18 primaries in the $\chi_{j=1, h}^{l} \bar{\chi}_{\bar{j}_{3}=-1}$ column can be organized into a A charge quintet and $\dot{A}$ charge triplet and a $A$ charge triplet and $\dot{A}$ charge singlet; thus the representations are $(2,1)$ and $(1,0)$.

For the lowest weight state of the $(2,1)$ multiplet, the normalized left movers of the long multiplet are

$$
\begin{aligned}
\left|\phi^{L}\right\rangle=\left|\phi_{+-}^{L}\right\rangle= & \frac{1}{4} \alpha_{+-,-1}^{(\mathcal{A})} \alpha_{+-,-1}^{(\mathcal{A})} d_{-1}^{--(\mathcal{A})} d_{0}^{+-(\mathcal{A})}\left|0_{R}^{-}\right\rangle\left|0_{R}^{-}\right\rangle \\
\left|\phi_{+}^{L}\right\rangle= & \frac{1}{2 \sqrt{6}}\left[\alpha_{+-,-3 / 2} \alpha_{++,-1 / 2} \alpha_{+-,-1 / 2} d_{-1 / 2}^{--}-\frac{1}{2} \alpha_{++,-3 / 2} \alpha_{+-,-1 / 2} \alpha_{+-,-1 / 2} d_{-1 / 2}^{--}\right. \\
& +\alpha_{+-,-1 / 2} d_{-3 / 2}^{--} d_{-1 / 2}^{+-} d_{-1 / 2}^{--}+\frac{1}{2} \alpha_{++,-1 / 2} \alpha_{+-,-1 / 2} \alpha_{+-,-1 / 2} d_{-3 / 2}^{--} \\
& -\frac{1}{2} \alpha_{++,-1 / 2} \alpha_{+-,-1 / 2} \alpha_{+-,-1 / 2} \alpha_{+-,-1 / 2} \alpha_{-+,-1 / 2} d_{-1 / 2}^{--} \\
& +\frac{1}{2} \alpha_{++,-1 / 2} \alpha_{++,-1 / 2} \alpha_{+-,-1 / 2} \alpha_{+-,-1 / 2} \alpha_{--,-1 / 2} d_{-1 / 2}^{--} \\
& \left.+\frac{1}{4} \alpha_{++,-1 / 2} \alpha_{+-,-1 / 2} \alpha_{+-,-1 / 2} d_{-1 / 2}^{+-} d_{-1 / 2}^{-+} d_{-1 / 2}^{--}\right]\left|0_{R}^{2-}\right\rangle \\
\left|\phi_{-}^{L}\right\rangle= & \frac{1}{2 \sqrt{6}}\left[\frac{1}{2} \alpha_{+-,-3 / 2} \alpha_{+-,-1 / 2} \alpha_{+-,-1 / 2} d_{-1 / 2}^{--}+\frac{1}{2} \alpha_{+-,-1 / 2} \alpha_{+-,-1 / 2} \alpha_{+-,-1 / 2} d_{-3 / 2}^{--}\right. \\
& -\frac{1}{2} \alpha_{+-,-1 / 2} \alpha_{+-,-1 / 2} \alpha_{+-,-1 / 2} \alpha_{+-,-1 / 2} \alpha_{-+,-1 / 2} d_{-1 / 2}^{--} \\
& +\frac{1}{2} \alpha_{++,-1 / 2} \alpha_{+-,-1 / 2} \alpha_{+-,-1 / 2} \alpha_{+-,-1 / 2} \alpha_{--,-1 / 2} d_{-1 / 2}^{--} \\
& \left.+\frac{1}{4} \alpha_{+-,-1 / 2} \alpha_{+-,-1 / 2} \alpha_{+-,-1 / 2} d_{-1 / 2}^{+-} d_{-1 / 2}^{-+} d_{-1 / 2}^{--}\right]\left|0_{R}^{2-}\right\rangle
\end{aligned}
$$

Applying (6.1) to the primary $\phi$, one finds the lift

$$
E_{3,(2,1)}^{(2)}=3 \pi^{2} \lambda^{2}
$$

For the lowest weight state of the $(1,0)$ multiplet, the normalized left movers of the long multiplet are

$$
\begin{aligned}
\left|\phi^{L}\right\rangle=\left|\phi_{+-}^{L}\right\rangle= & \frac{1}{2 \sqrt{46}}\left[d_{-3}^{--(\mathcal{A})} d_{0}^{+-(\mathcal{A})}-2 d_{-2}^{--(\mathcal{A})} d_{-1}^{+-(\mathcal{A})}-d_{-2}^{+-(\mathcal{A})} d_{-1}^{--(\mathcal{A})}\right. \\
& +\frac{3}{2} d_{-2}^{--(\mathcal{A})} d_{-1}^{--(\mathcal{A})} d_{0}^{++(\mathcal{A})} d_{0}^{+-(\mathcal{A})}-\frac{3}{2} d_{-1}^{+-(\mathcal{A})} d_{-1}^{--(\mathcal{A})} d_{-1}^{-+(\mathcal{A})} d_{0}^{+-(\mathcal{A})} \\
& +\frac{3}{2}\left(\alpha_{++,-1}^{(\mathcal{A})} \alpha_{--,-1}^{(\mathcal{A})}-\alpha_{+-,-1}^{(A)} \alpha_{-+,-1}^{(\mathcal{A})} d_{-1}^{--(\mathcal{A})} d_{0}^{+-(\mathcal{A})}\right. \\
& \left.+\alpha_{++,-2}^{(\mathcal{A})} \alpha_{+-,-1}^{(\mathcal{A})}-\alpha_{+-,-2}^{(\mathcal{A})} \alpha_{++,-1}^{(\mathcal{A})}\right]\left|0_{R}^{-}\right\rangle\left|0_{R}^{-}\right\rangle \\
\left|\phi_{+}^{L}\right\rangle= & \frac{1}{\sqrt{897}}\left[\frac{15}{16} \alpha_{++,-5 / 2} d_{-1 / 2}^{--}-\frac{29}{8} \alpha_{++,-3 / 2} d_{-3 / 2}^{--}+\frac{75}{16} \alpha_{++,-1 / 2} d_{-5 / 2}^{--}\right. \\
& +\frac{27}{16} \alpha_{--,-3 / 2} \alpha_{++,-1 / 2} \alpha_{++,-1 / 2} d_{-1 / 2}^{--}-\frac{27}{16} \alpha_{-+,-3 / 2} \alpha_{++,-1 / 2} \alpha_{+-,-1 / 2} d_{-1 / 2}^{--}
\end{aligned}
$$




$$
\begin{aligned}
& -\frac{43}{16} \alpha_{+-,-3 / 2} \alpha_{++,-1 / 2} \alpha_{-+,-1 / 2} d_{-1 / 2}^{--}-\alpha_{+-,-3 / 2} \alpha_{++,-1 / 2} \alpha_{++,-1 / 2} d_{-1 / 2}^{-+} \\
& +\frac{21}{8} \alpha_{++,-3 / 2} \alpha_{+-,-1 / 2} \alpha_{-+,-1 / 2} d_{-1 / 2}^{--}+\frac{1}{16} \alpha_{++,-3 / 2} \alpha_{++,-1 / 2} \alpha_{--,-1 / 2} d_{-1 / 2}^{--} \\
& +\alpha_{++,-3 / 2} \alpha_{++,-1 / 2} \alpha_{+-,-1 / 2} d_{-1 / 2}^{-+}-\frac{21}{8} \alpha_{++,-1 / 2} \alpha_{+-,-1 / 2} \alpha_{-+,-1 / 2} d_{-3 / 2}^{--} \\
& +\frac{21}{8} \alpha_{++,-1 / 2} \alpha_{++,-1 / 2} \alpha_{--,-1 / 2} d_{-3 / 2}^{--}-\frac{29}{16} \alpha_{++,-3 / 2} d_{-1 / 2}^{+-} d_{-1 / 2}^{-+} d_{-1 / 2}^{--} \\
& -\frac{21}{4} \alpha_{-+,-1 / 2} d_{-3 / 2}^{--} d_{-1 / 2}^{+-} d_{-1 / 2}^{--}-\frac{3}{16} \alpha_{++,-1 / 2} d_{-3 / 2}^{--} d_{-1 / 2}^{+-} d_{-1 / 2}^{-+} \\
& -\frac{81}{32} \alpha_{++,-1 / 2} d_{-3 / 2}^{--} d_{-1 / 2}^{++} d_{-1 / 2}^{--}-\frac{81}{32} \alpha_{++,-1 / 2} d_{-3 / 2}^{-+} d_{-1 / 2}^{+-} d_{-1 / 2}^{--} \\
& +\frac{75}{32} \alpha_{++,-1 / 2} d_{-3 / 2}^{+-} d_{-1 / 2}^{-+} d_{-1 / 2}^{--} \\
& -\frac{21}{16} \alpha_{++,-1 / 2} \alpha_{+-,-1 / 2} \alpha_{-+,-1 / 2} d_{-1 / 2}^{+-} d_{-1 / 2}^{-+} d_{-1 / 2}^{--} \\
& +\frac{21}{16} \alpha_{++,-1 / 2} \alpha_{++,-1 / 2} \alpha_{--,-1 / 2} d_{-1 / 2}^{+-} d_{-1 / 2}^{-+} d_{-1 / 2}^{--} \\
& +\frac{21}{16} \alpha_{++,-1 / 2} \alpha_{+-,-1 / 2} \alpha_{+-,-1 / 2} \alpha_{-+,-1 / 2} \alpha_{-+,-1 / 2} d_{-1 / 2}^{--} \\
& -\frac{21}{8} \alpha_{++,-1 / 2} \alpha_{++,-1 / 2} \alpha_{+-,-1 / 2} \alpha_{-+,-1 / 2} \alpha_{--,-1 / 2} d_{-1 / 2}^{--} \\
& \left.+\frac{21}{16} \alpha_{++,-1 / 2} \alpha_{++,-1 / 2} \alpha_{++,-1 / 2} \alpha_{--,-1 / 2} \alpha_{--,-1 / 2} d_{-1 / 2}^{--}\right]\left|0_{R}^{2-}\right\rangle
\end{aligned}
$$

Because $\phi^{L}$ is a $\dot{A}$ charge singlet, $\phi_{-}^{L}$ is given by doing the following replacement in $\phi_{+}^{L}$.

$$
\alpha_{A \pm, n} \rightarrow i \alpha_{A \mp, n}
$$

Applying (6.1) to the primary $\phi$, one finds the lift

$$
E_{3,(1,0)}^{(2)}=\frac{64}{39} \pi^{2} \lambda^{2}
$$

\subsection{Level-4}

The 15 primaries in the $\chi_{j=1, h}^{l} \bar{\chi}_{\bar{j}_{3}=-1}$ column can be organized into a $A$ charge triplet and $\dot{A}$ charge triplet $(1,1)$, a $A$ charge quintet and $\dot{A}$ charge singlet $(2,0)$ and a $A$ charge singlet and $\dot{A}$ charge singlet $(0,0)$. Their lifts are

$$
\begin{aligned}
& E_{4,(1,1)}^{(2)}=3 \pi^{2} \lambda^{2} \\
& E_{4,(2,0)}^{(2)}=\frac{5}{2} \pi^{2} \lambda^{2} \\
& E_{4,(0,0)}^{(2)}=\frac{2695}{1024} \pi^{2} \lambda^{2}
\end{aligned}
$$

Since the states are very complicated, we will not write them explicitly here. 


\subsection{Properties of lifting at $O\left(\lambda^{2}\right)$}

In this subsection, we summarize the properties of the lift at $O\left(\lambda^{2}\right)$ for primaries in the theory with total winding $N=2$.

(i) From the index, we expect that primaries in the columns $\chi_{j=1, h}^{l} \bar{\chi}_{\bar{j}_{3}=-1}$ and $\chi_{j=1, h}^{l} \bar{\chi}_{\bar{j}_{3}=0}$ are lifted. At order $O\left(\lambda^{2}\right)$, we find that primaries up to level-4 are all lifted. They are the states $\phi$ and $\phi_{+-}$in the long multiplet (3.7).

From eq. (5.1) we see that the right movers of primaries in these two columns are symmetric between the two copies. In the orbifold CFT the overall state of each string must be symmetric between the copies. Thus primaries in these two columns must be symmetric between the copies in both the left and right sectors separately.

(ii) From the index, we expect that primaries in the column $\chi_{j=1, h}^{l} \bar{\chi}_{\bar{j}_{3}=-1 / 2}$ in the $(1,1)$ sector are unlifted. At order $O\left(\lambda^{2}\right)$, we find that primaries at any level in this column are unlifted. The reason is the following. The right moving state is (5.2), which is antisymmetric between the two copies. Thus primaries in this column are antisymmetric in the left and right sectors separately. Because the projection operator (3.5) treats the left mover and right mover of the two copies symmetrically in the lifting computation, antisymmetry in both the left and right sectors leads to zero lift.

(iii) The lifting properties of primaries in the (2) sector determined by the details of operator (3.5). Because number of primaries in the (2) sector grows faster than the numbers of primaries in the columns $\chi_{j=1, h}^{l} \bar{\chi}_{\bar{j}_{3}=-1}$ and $\chi_{j=1, h}^{l} \bar{\chi}_{\bar{j}_{3}=0}$ in the $(1,1)$ sector, most of the primaries in the (2) sector will be unlifted. The lifted states are the $\phi_{+}$and $\phi_{-}$in the long multiplet (3.7).

\section{Unlifted states in the untwisted sector}

In section 6 , we found that in our study upto level-4, the unlifted states in the $(1,1)$ sector are states that have a right moving sector that is antisymmetric between the two copies. In this section, we will study the consequences of this antisymmetry in more detail, and generalize it to the situation where the component strings have higher twists.

In section 7.1, we study the antisymmetry of the right movers for two singly wound strings. In section 7.2 , we generalize this antisymmetry to the situation where we have many singly wound strings. In section 7.3 , we generalize this discussion to the case where we have two component strings with winding numbers $k_{1}$ and $k_{2}$.

\subsection{Right movers for the $(1,1)$ sector}

Consider the case where $N=2$ and we have two singly wound strings. Let us focus on the right movers. We have used the fact that we can write the right movers on the two strings using the sum and difference of the oscillators on the two strings. Thus we get the global modes

$$
\bar{d}_{0}^{+A(1)}+\bar{d}_{0}^{+A(2)}
$$


which are symmetric between the two copies, and the antisymmetric modes

$$
\bar{d}_{0}^{+A(1)}-\bar{d}_{0}^{+A(2)}
$$

which are antisymmetric between the two copies. We have seen that states where the right moving sector contains one antisymmetric mode are unlifted, while those containing zero or two antisymmetric modes are lifted. Thus unlifted states have an antisymmetric right sector while lifted states have a symmetric right sector. For example, the right moving states

$$
\bar{d}_{0}^{+ \pm(1)} \bar{d}_{0}^{+ \pm(2)}\left|\overline{0}_{R}^{-}\right\rangle^{(1)}\left|\overline{0}_{R}^{-}\right\rangle^{(2)}=\frac{1}{2}\left(\bar{d}_{0}^{+ \pm(1)} \bar{d}_{0}^{+ \pm(2)}-\bar{d}_{0}^{+ \pm(2)} \bar{d}_{0}^{+ \pm(1)}\right)\left|\overline{0}_{R}^{-}\right\rangle^{(1)}\left|\overline{0}_{R}^{-}\right\rangle^{(2)}
$$

and

$$
\left(\bar{d}_{0}^{++(1)} \bar{d}_{0}^{+-(2)}-\bar{d}_{0}^{++(2)} \bar{d}_{0}^{+-(1)}\right)\left|\overline{0}_{R}^{-}\right\rangle^{(1)}\left|\overline{0}_{R}^{-}\right\rangle^{(2)}
$$

are antisymmetric and thus lead to zero lift.

\subsection{More general states}

Let us now consider the case $N>2$. We will see that we can use the above notion of antisymmetrization of the right sector to make a large class of states that are unlifted at $O\left(\lambda^{2}\right)$.

We first illustrate the idea by a simple example. Consider 3 singly wound strings. Let the left and right sectors of each string be fermionic, so that each string is overall in a bosonic state. Let the left sides of each string be in a different excited state:

$$
\left|\psi_{L}^{1}\right\rangle, \quad\left|\psi_{L}^{2}\right\rangle, \quad\left|\psi_{L}^{3}\right\rangle
$$

Let the right sides be given by

$$
\bar{d}_{0}^{+A_{1}(1)}\left|\overline{0}_{R}^{-}\right\rangle^{(1)}, \quad \bar{d}_{0}^{+A_{2}(2)}\left|\overline{0}_{R}^{-}\right\rangle^{(2)}, \quad \bar{d}_{0}^{+A_{3}(3)}\left|\overline{0}_{R}^{-}\right\rangle^{(3)}
$$

Thus the overall state is

$$
|\Psi\rangle=-\left[\bar{d}_{0}^{+A_{1}(1)} \bar{d}_{0}^{+A_{2}(2)} \bar{d}_{0}^{+A_{3}(3)}\right]\left|\psi_{L}^{1}\right\rangle\left|\overline{0}_{R}^{-}\right\rangle^{(1)}\left|\psi_{L}^{2}\right\rangle\left|\overline{0}_{R}^{-}\right\rangle^{(2)}\left|\psi_{L}^{3}\right\rangle\left|\overline{0}_{R}^{-}\right\rangle^{(3)}
$$

We now look at different cases

(i) All $A_{i}$ are the same: let us set $A_{i}=+$ for all $i$. Thus the state is

$$
|\Psi\rangle=-\left[\bar{d}_{0}^{++(1)} \bar{d}_{0}^{++(2)} \bar{d}_{0}^{++(3)}\right]\left|\psi_{L}^{1}\right\rangle\left|\overline{0}_{R}^{-}\right\rangle^{(1)}\left|\psi_{L}^{2}\right\rangle\left|\overline{0}_{R}^{-}\right\rangle^{(2)}\left|\psi_{L}^{3}\right\rangle\left|\overline{0}_{R}^{-}\right\rangle^{(3)}
$$

As shown in (7.3), the right mover is antisymmetric between any two copies. If we twist any two strands together, then we get zero, so this state is unlifted to $O\left(\lambda^{2}\right)$.

(ii) One $A_{i}$ is different: let us set $A_{1}=+, A_{2}=+, A_{3}=-$. The state is

$$
\left|\Psi_{1}\right\rangle=-\left[\bar{d}_{0}^{++(1)} \bar{d}_{0}^{++(2)} \bar{d}_{0}^{+-(3)}\right]\left|\psi_{L}^{1}\right\rangle\left|\overline{0}_{R}^{-}\right\rangle^{(1)}\left|\psi_{L}^{2}\right\rangle\left|\overline{0}_{R}^{-}\right\rangle^{(2)}\left|\psi_{L}^{3}\right\rangle\left|\overline{0}_{R}^{-}\right\rangle^{(3)}
$$


There is no lift if we twist together strands 1,2. But there is a lift for strands 2,3. Thus let us take instead the antisymmetrized state

$$
\begin{aligned}
&\left|\Psi_{2}\right\rangle=-\left[\bar{d}_{0}^{++(1)} \bar{d}_{0}^{++(2)} \bar{d}_{0}^{+-(3)}-\bar{d}_{0}^{++(1)} \bar{d}_{0}^{++(3)} \bar{d}_{0}^{+-(2)}-\bar{d}_{0}^{++(3)} \bar{d}_{0}^{++(2)} \bar{d}_{0}^{+-(1)}\right] \\
&\left|\psi_{L}^{1}\right\rangle\left|\overline{0}_{R}^{-}\right\rangle^{(1)}\left|\psi_{L}^{2}\right\rangle\left|\overline{0}_{R}^{-}\right\rangle^{(2)}\left|\psi_{L}^{3}\right\rangle\left|\overline{0}_{R}^{-}\right\rangle^{(3)} \\
&=-\left[\bar{d}_{0}^{++(1)} \bar{d}_{0}^{++(2)} \bar{d}_{0}^{+-(3)}+\bar{d}_{0}^{++(1)} \bar{d}_{0}^{+-(2)} \bar{d}_{0}^{++(3)}+\bar{d}_{0}^{+-(1)} \bar{d}_{0}^{++(2)} \bar{d}_{0}^{++(3)}\right] \\
&\left|\psi_{L}^{1}\right\rangle\left|\overline{0}_{R}^{-}\right\rangle^{(1)}\left|\psi_{L}^{2}\right\rangle\left|\overline{0}_{R}^{-}\right\rangle^{(2)}\left|\psi_{L}^{3}\right\rangle\left|\overline{0}_{R}^{-}\right\rangle^{(3)}
\end{aligned}
$$

Now we find that there is no lift at $O\left(\lambda^{2}\right)$ from twisting any pair of strings.

We can now see that the above example is easily generalized. We can take any set of states with arbitrary left excitations, and antisymmetrize the right movers. This will make the states unlifted at $O\left(\lambda^{2}\right)$. Note that when two strings have the same bosonic right movers, their antisymmetrization will lead to a vanishing of the state. Thus this method of making unlifted states can only be used when we have fermionic right movers.

\subsection{Strings with winding numbers $k_{1}$ and $k_{2}$}

In section 7.1, we had considered the case where two singly wound strings joined to a doubly wound string. In this subsection, we consider the analogous problem for the situation where a string with winding $k_{1}$ and a string with winding $k_{2}$ join to a string with winding $k_{1}+k_{2}$.

Right movers in the $\left(k_{1}, k_{2}\right)$ sector can be built by the global modes

$$
\bar{d}_{0}^{+A\left(k_{1}\right)}+\bar{d}_{0}^{+A\left(k_{2}\right)}
$$

and the modes

$$
\frac{1}{k_{1}} \bar{d}_{0}^{+A\left(k_{1}\right)}-\frac{1}{k_{2}} \bar{d}_{0}^{+A\left(k_{2}\right)}
$$

acting on the ground state

$$
\left|\overline{0}_{R}^{k_{1}-}\right\rangle\left|\overline{0}_{R}^{k_{2}-}\right\rangle
$$

The global modes (7.11) do not contribute to the lift. In appendix D, we find that the operators $\bar{G}_{\dot{A}, 0}^{\bar{\alpha}(P)}$ annihilate the right moving states containing one operator of type (7.12); the operators $\bar{G}_{\dot{A}, 0}^{\bar{\alpha}(P)}$ do not annihilate the right moving states containing zero or two operators of $(7.12)$.

Thus the contribution to the lift from the interaction joining together strings with winding numbers $k_{1}$ and $k_{2}$ is zero for states with right movers containing one operator of (7.12).

Note that the twist interaction can also break the strings of winding $k_{1}$ or $k_{2}$ into shorter strings. But we do not consider this effect here; if the windings $k_{1}, k_{2}$ are much smaller than the total number $N$ of component strings, then combinatorical factors suppress the interactions where one strand of the string twists together with another strand of the same string.

The modes (7.11) and (7.12) are the analogues of the symmetric and antisymmetric modes (7.1) and (7.2). However, they do not have definite symmetry properties. 
Now let us consider states with a fermionic right moving sector for each string. Consider the right moving states

$$
\begin{aligned}
& \bar{d}_{0}^{+ \pm\left(k_{1}\right)} \bar{d}_{0}^{+ \pm\left(k_{2}\right)}\left|\overline{0}_{R}^{k_{1}-}\right\rangle\left|\overline{0}_{R}^{k_{2}-}\right\rangle \\
& \quad=-\frac{k_{1} k_{2}}{k_{1}+k_{2}}\left(\bar{d}_{0}^{+ \pm\left(k_{1}\right)}+\bar{d}_{0}^{+ \pm\left(k_{2}\right)}\right)\left(\frac{1}{k_{1}} \bar{d}_{0}^{+ \pm\left(k_{1}\right)}-\frac{1}{k_{2}} \bar{d}_{0}^{+ \pm\left(k_{2}\right)}\right)\left|\overline{0}_{R}^{k_{1}-}\right\rangle\left|\overline{0}_{R}^{k_{2}-}\right\rangle
\end{aligned}
$$

and

$$
\begin{aligned}
& \bar{d}_{0}^{++\left(k_{1}\right)} \bar{d}_{0}^{+-\left(k_{2}\right)}-\bar{d}_{0}^{++\left(k_{2}\right)} \bar{d}_{0}^{+-\left(k_{1}\right)} \\
&=-\frac{k_{1} k_{2}}{k_{1}+k_{2}} {\left[\left(\bar{d}_{0}^{++\left(k_{1}\right)}+\bar{d}_{0}^{++\left(k_{2}\right)}\right)\left(\frac{1}{k_{1}} \bar{d}_{0}^{+-\left(k_{1}\right)}-\frac{1}{k_{2}} \bar{d}_{0}^{+-\left(k_{2}\right)}\right)\right.} \\
&\left.\quad+\left(\bar{d}_{0}^{+-\left(k_{1}\right)}+\bar{d}_{0}^{+-\left(k_{2}\right)}\right)\left(\frac{1}{k_{1}} \bar{d}_{0}^{++\left(k_{1}\right)}-\frac{1}{k_{2}} \bar{d}_{0}^{++\left(k_{2}\right)}\right)\right]\left|\overline{0}_{R}^{k_{1}-}\right\rangle\left|\overline{0}_{R}^{k_{2}-}\right\rangle
\end{aligned}
$$

States with right movers (7.14) and (7.15) will be unlifted because each term contains only one mode of (7.12). Note that these two right moving states are antisymmetric under the change $k_{1} \leftrightarrow k_{2}$. If we consider other possible right moving states that lead to zero lift, then we find that there is no definite symmetric property of such states in general; what we have noted here is that in the case where we have fermionic right movers for each string the state is antisymmetric under the change $k_{1} \leftrightarrow k_{2}$.

Thus, we can generalize the discussion in subsection 7.2. We take states with arbitrary winding numbers, arbitrary left excitations and only fermionic right movers. Antisymmetrizing the fermionic right movers will make the states unlifted at $O\left(\lambda^{2}\right)$, in the approximation where we only consider the effect of twisting two strings into a longer string and ignore the breaking of the strings to shorter strings.

\section{Discussion}

To understand the AdS/CFT correspondence we need to match quantities between the gravity and field theory descriptions. The principal difficulty in this match is the fact that one of these descriptions is weakly coupled, then the other is strongly coupled. There have been much progress in understanding black holes by comparing quantities in the free CFT to results with weakly coupled gravity; however a more detailed comparison will necessarily need us to perturb the CFT away from its free (i.e. orbifold) point. The most basic quantity we can study under such a perturbation is the energy levels of states in the CFT as a function of the perturbation parameter $\lambda$.

In this paper we have considered states which are BPS at the orbifold point, and asked which states are lifted and by how much as we perturb away from this point. For most of this paper we worked with the simplest system which allows a computation of the lift: the case $N=2$ where we have 2 copies of the basic $c=6$ CFT making up the orbifold theory. The lifted states must join into a supermultiplet in which all members will have the same lift; we found this multiplet structure and the value of the lift to order $O\left(\lambda^{2}\right)$ for the first 4 levels above the Ramond ground state. For the first 3 levels we also explicitly write down 
the wavefunctions of the lifted states. We found that all the states that are allowed to lift by the index are in fact lifted at $O\left(\lambda^{2}\right)$.

We observed that the unlifted states in the untwisted sector have a right moving sector which was antisymmetric between the two copies of the $c=6 \mathrm{CFT}$. We then extended this observation to the case $N>2$, where we found states which would remain unlifted to order $O\left(\lambda^{2}\right)$ by using a feature similar to this antisymmetry.

For large $N$, one can consider the gravity dual. In this gravity description a large class of supergravity states called 'superstrata' were found in [52-57]. These are extremal states that describe fully backreacted solutions arising from massless string quanta placed in the $A d S_{3} \times S^{3} \times T^{4}$ geometry. The analysis of lifting carried out in the present paper may help us to understand the nature of the CFT states that are dual to these BPS gravity solutions. We hope to return to this issue in a future work.

\section{Acknowledgments}

We would like to thank Nathan Benjamin, Stefano Giusto, Shaun Hampton, Rodolfo Russo, David Turton, Ida Zadeh and Xinan Zhou, for many helpful discussions. This work is supported in part by DOE grant de-sc0011726.

\section{A Contracted large $\mathcal{N}=4$ superconformal algebra}

We follow the notation in the appendix of [45]. The indices $\alpha=(+,-)$ and $\bar{\alpha}=(+,-)$ correspond to the subgroups $\mathrm{SU}(2)_{L}$ and $\mathrm{SU}(2)_{R}$ arising from rotations on $S^{3}$. The indices $A=(+,-)$ and $\dot{A}=(+,-)$ correspond to the subgroups $\mathrm{SU}(2)_{1}$ and $\mathrm{SU}(2)_{2}$ arising from rotations in $T^{4}$. We use the convention

$$
\epsilon_{+-}=1, \quad \epsilon^{+-}=-1
$$

The commutation relations for the contracted large $\mathcal{N}=4$ superconformal algebra are

$$
\begin{aligned}
{\left[\alpha_{A \dot{A}, m}, \alpha_{B \dot{B}, n}\right] } & =-\frac{c}{6} m \epsilon_{A B} \epsilon_{\dot{A} \dot{B}} \delta_{m+n, 0} \\
\left\{d_{r}^{\alpha A}, d_{s}^{\beta B}\right\} & =-\frac{c}{6} \epsilon^{\alpha \beta} \epsilon^{A B} \delta_{r+s, 0} \\
{\left[L_{m}, \alpha_{A \dot{A}, n}\right] } & =-n \alpha_{A \dot{A}, m+n} \quad\left[L_{m}, d_{r}^{\alpha A}\right]=-\left(\frac{m}{2}+r\right) d_{m+r}^{\alpha A} \\
\left\{G_{\dot{A}, r}^{\alpha}, d_{s}^{\beta B}\right\} & =i \epsilon^{\alpha \beta} \epsilon^{A B} \alpha_{A \dot{A}, r+s} \quad\left[G_{\dot{A}, r}^{\alpha}, \alpha_{B \dot{B}, m}\right]=-i m \epsilon_{A B} \epsilon_{\dot{A} \dot{B}} d_{r+m}^{\alpha A} \\
{\left[J_{m}^{a}, d_{r}^{\alpha A}\right] } & =\frac{1}{2}\left(\sigma^{T a}\right)_{\beta}^{\alpha} d_{m+r}^{\beta A} \\
{\left[L_{m}, L_{n}\right] } & =\frac{c}{12} m\left(m^{2}-1\right) \delta_{m+n, 0}+(m-n) L_{m+n} \\
{\left[J_{m}^{a}, J_{n}^{b}\right] } & =\frac{c}{12} m \delta^{a b} \delta_{m+n, 0}+i \epsilon_{c}^{a b} J_{m+n}^{c} \\
\left\{G_{\dot{A}, r}^{\alpha}, G_{\dot{B}, s}^{\beta}\right\} & =\epsilon_{\dot{A} \dot{B}}\left[\epsilon^{\alpha \beta} \frac{c}{6}\left(r^{2}-\frac{1}{4}\right) \delta_{r+s, 0}+\left(\sigma^{a T}\right)_{\gamma}^{\alpha} \epsilon^{\gamma \beta}(r-s) J_{r+s}^{a}+\epsilon^{\alpha \beta} L_{r+s}\right]
\end{aligned}
$$




$$
\begin{aligned}
{\left[J_{m}^{a}, G_{\dot{A}, r}^{\alpha}\right] } & =\frac{1}{2}\left(\sigma^{a T}\right)_{\beta}^{\alpha} G_{\dot{A}, m+r}^{\beta} \\
{\left[L_{m}, J_{n}^{a}\right] } & =-n J_{m+n}^{a} \\
{\left[L_{m}, G_{\dot{A}, r}^{\alpha}\right] } & =\left(\frac{m}{2}-r\right) G_{\dot{A}, m+r}^{\alpha}
\end{aligned}
$$

We define $J_{n}^{+}, J_{n}^{-}$as

$$
\begin{aligned}
& J_{n}^{+}=J_{n}^{1}+i J_{n}^{2} \\
& J_{n}^{-}=J_{n}^{1}-i J_{n}^{2}
\end{aligned}
$$

From (A.3), one can see that $d_{n}^{\alpha A}$ with $\alpha=+,-$ is a $\mathrm{SU}(2)_{L}$ charge doublet. We have

$$
\begin{array}{lll}
{\left[J_{m}^{+}, d_{r}^{+A}\right]=0,} & {\left[J_{m}^{-}, d_{r}^{+A}\right]=d_{m+r}^{-A}} \\
{\left[J_{m}^{+}, d_{r}^{-A}\right]=d_{m+r}^{+A},} & {\left[J_{m}^{-}, d_{r}^{-A}\right]=0}
\end{array}
$$

From (A.4), one can see that $G_{\dot{A}, r}^{\alpha}$ with $\alpha=+,-$ is also a $\mathrm{SU}(2)_{L}$ charge doublet. We have

$$
\begin{array}{lll}
{\left[J_{m}^{+}, G_{\dot{A}, r}^{+}\right]=0,} & {\left[J_{m}^{-}, G_{\dot{A}, r}^{+}\right]=G_{\dot{A}, m+r}^{-}} \\
{\left[J_{m}^{+}, G_{\dot{A}, r}^{-}\right]=G_{\dot{A}, m+r}^{+},} & {\left[J_{m}^{-}, G_{\dot{A}, r}^{-}\right]=0}
\end{array}
$$

It is believed that the contracted large $\mathcal{N}=4$ superconformal algebra is an exact symmetry at any point of the moduli space.

Now let's consider the orbifold point. Look at the winding sector $\left(k_{1}, k_{2}, \ldots, k_{i}, \ldots\right)$ with the total winding $N=\sum_{i} k_{i}$. For the $i$ th twisted set of copies with winding number $k_{i}$, we have following mode expansions on the cylinder.

$$
\begin{aligned}
\alpha_{A \dot{A}, n}^{(i)} & =\frac{1}{2 \pi} \int_{\sigma=0}^{2 \pi k_{i}} \partial_{w} X_{A \dot{A}}^{(i)}(w) e^{n w} d w \\
d_{n}^{\alpha A(i)} & =\frac{1}{2 \pi i} \int_{\sigma=0}^{2 \pi k_{i}} \psi^{\alpha A(i)}(w) e^{n w} d w
\end{aligned}
$$

In terms of $\alpha$ and $d$ modes, the $J, G$ and $L$ modes can be written as

$$
\begin{aligned}
J_{m}^{a(i)} & =\frac{1}{4 k_{i}} \sum_{r} \epsilon_{A B} d_{r}^{\gamma B(i)} \epsilon_{\alpha \gamma}\left(\sigma^{a T}\right)_{\beta}^{\alpha} d_{m-r}^{\beta A(i)}, \quad a=1,2,3 \\
J_{m}^{3(i)} & =-\frac{1}{2 k_{i}} \sum_{r} d_{r}^{++(i)} d_{m-r}^{--(i)}-\frac{1}{2 k_{i}} \sum_{r} d_{r}^{-+(i)} d_{m-r}^{+-(i)} \\
J_{m}^{+(i)} & =\frac{1}{k_{i}} \sum_{r} d_{r}^{++(i)} d_{m-r}^{+-(i)}, \quad J_{m}^{-(i)}=\frac{1}{k_{i}} \sum_{r} d_{r}^{--(i)} d_{m-r}^{-+(i)} \\
G_{\dot{A}, r}^{\alpha(i)} & =-\frac{i}{k_{i}} \sum_{n} d_{r-n}^{\alpha A(i)} \alpha_{A \dot{A}, n}^{(i)} \\
L_{m}^{(i)} & =-\frac{1}{2 k_{i}} \sum_{n} \epsilon^{A B} \epsilon^{\dot{A} \dot{B}} \alpha_{A \dot{A}, n}^{(i)} \alpha_{B \dot{B}, m-n}^{(i)}-\frac{1}{2 k_{i}} \sum_{r}\left(m-r+\frac{1}{2}\right) \epsilon_{\alpha \beta} \epsilon_{A B} d_{r}^{\alpha A(i)} d_{m-r}^{\beta B(i)}
\end{aligned}
$$


Let $q$ be an integer. The mode numbers for $\alpha, L, J$ are $n=q / k_{i}$. In the $\mathrm{R}$ sector, the mode numbers for $d$ and $G$ are $n=q / k_{i}$. In the NS sector, the mode numbers for $d$ and $G$ are $n=\left(q+\frac{1}{2}\right) / k_{i}$. The modes (A.8) and (A.9) satisfy the contracted large $\mathcal{N}=4$ superconformal algebra (A.2)(A.3)(A.4) with $c=6 k_{i}$.

We define the global modes $O_{n}^{(g)}$ by summing the terms from each copy

$$
O_{n}^{(g)}=\sum_{i} O_{n}^{(i)}
$$

where the modes $O$ can be modes of $\alpha, d, L, J, G$. The global modes satisfy the contracted large $\mathcal{N}=4$ superconformal algebra (A.2)(A.3)(A.4) with $c=6 N$. It is believed that global modes satisfy the algebra at any point in the moduli space.

\section{B The $\mathcal{N}=4$ character}

In this appendix, we will present the character of the small and the contracted large $\mathcal{N}=4$ superconformal algebra. The characters are defined as follows:

$$
\chi_{j, h}(q, y)=\operatorname{Tr}(-1)^{2 J_{0}^{3}} q^{L_{0}-\frac{c}{24}} y^{2 J_{0}^{3}}
$$

where the trace is over states in the irreducible representation. In this paper we will work in the Ramond sector. The primaries in these represenations have dimension $h$, which is the eigenvalue of $L_{0}-\frac{c}{24}$, and $\mathrm{SU}(2)$ charge $j$.

\section{B.1 The small $\mathcal{N}=4$ character}

The characters were computed in $[58,59]$. The central charge is given by $c=6 m$, where $m$ is an integer. We use $\chi^{(S)}$ to label the character in the small $\mathcal{N}=4$ algebra. There are $m+1$ short representations with $h=0$ and $j=0,1 / 2, \ldots, m / 2$. Their characters are

$$
\begin{aligned}
\chi_{j ; m}^{s(S)}(\tau, z)= & (-1)^{2 j}\left(\frac{i \theta_{1}(\tau, z)^{2}}{\theta_{1}(\tau, 2 z) \eta(\tau)^{3}}\right) \\
& \sum_{k \in \mathbb{Z}} \frac{q^{(m+1) k^{2}+k} y^{2(m+1) k+1}}{\left(1-y q^{k}\right)^{2}}\left(q^{k(2 j+1)} y^{2 j+1}-q^{-k(2 j+1)} y^{-(2 j+1)}\right)
\end{aligned}
$$

where we define $q=e^{2 \pi i \tau}$ and $y=e^{2 \pi i z}$. There are $m$ long representations with $h>0$ and $j=1 / 2,1, \ldots, m / 2$. Their characters are

$$
\chi_{j, h ; m}^{l(S)}(\tau, z)=q^{h}(-1)^{2 j}\left(\frac{i \theta_{1}(\tau, z)^{2}}{\theta_{1}(\tau, 2 z) \eta(\tau)^{3}}\right) \sum_{k \in \mathbb{Z}} q^{(m+1) k^{2}} y^{2(m+1) k}\left(q^{2 k j} y^{2 j}-q^{-2 k j} y^{-2 j}\right)
$$

\section{B.2 The contracted large $\mathcal{N}=4$ character}

The characters were computed in [60,61]. The central charge is given by $c=6 m$, where $m$ is an integer. There are $m$ short representations with $h=0$ and $j=1 / 2,1, \ldots, m / 2$

$$
\chi_{j ; m}^{s}(\tau, z)=\frac{q^{1 / 8} L(\tau, z)}{\eta(\tau)^{3}} \chi_{j-1 / 2 ; m-1}^{s(S)}(\tau, z)
$$


There are $m-1$ long representations with $h>0$ and $j=1, \ldots, m / 2$

$$
\chi_{j, h ; m}^{l}(\tau, z)=\frac{q^{1 / 8} L(\tau, z)}{\eta(\tau)^{3}} \chi_{j-1 / 2, h ; m-1}^{l(S)}(\tau, z)
$$

where

$$
L(\tau, z)=\chi_{j=1 / 2, h=0 ; m=1}^{l(S)}(\tau, z)
$$

Here $\chi^{s(S)}$ and $\chi^{l(S)}$ are characters of the short and long multiplets (B.2)(B.3) of the small $\mathcal{N}=4$ algebra.

\section{The effect of the twist operator}

The operator (3.5) contains the twist operator $\sigma_{2}$. The action of this twist was studied in $[21,22]$. Here we recall some results about this action which will be of use to us later in the computation of $E^{(2)}$.

We consider only the left sector. Start in the twist sector $N=(1,1)$ where we have two singly wound copies of the CFT. Let the initial state be the Ramond ground state $\left|0_{R}^{-}\right\rangle\left|0_{R}^{-}\right\rangle$. Let us apply the twist operator $\sigma_{2}^{+}$at the position $w_{0}$ on the cylinder. This action generates the state $|\chi\rangle$

$$
\begin{aligned}
|\chi\rangle=\sigma_{2}^{+}\left(w_{0}\right)\left|0_{R}^{-}\right\rangle\left|0_{R}^{-}\right\rangle= & e^{\sum_{m \geq 1 / 2, n \geq 1 / 2} \gamma_{m n}^{B}\left[-\alpha_{++,-m} \alpha_{--,-n}+\alpha_{-+,-m} \alpha_{+-,-n}\right]} \\
& e^{\sum_{m \geq 1 / 2, n \geq 1 / 2} \gamma_{m n}^{F}\left[d_{-m}^{++} d_{-n}^{--}-d_{-m}^{+-} d_{-n}^{-+}\right]}\left|0_{R}^{2-}\right\rangle
\end{aligned}
$$

where

$$
\begin{aligned}
\gamma_{m^{\prime}+1 / 2, n^{\prime}+1 / 2}^{B} & =\frac{2}{\left(2 m^{\prime}+1\right)\left(2 n^{\prime}+1\right)} \frac{a^{2\left(m^{\prime}+n^{\prime}+1\right)} \Gamma\left[\frac{3}{2}+m^{\prime}\right] \Gamma\left[\frac{3}{2}+n^{\prime}\right]}{\left(1+m^{\prime}+n^{\prime}\right) \pi \Gamma\left[m^{\prime}+1\right] \Gamma\left[n^{\prime}+1\right]} \\
\gamma_{m^{\prime}+1 / 2, n^{\prime}+1 / 2}^{F} & =-\frac{a^{2\left(m^{\prime}+n^{\prime}+1\right)} \Gamma\left[\frac{3}{2}+m^{\prime}\right] \Gamma\left[\frac{3}{2}+n^{\prime}\right]}{\left(2 n^{\prime}+1\right) \pi\left(1+m^{\prime}+n^{\prime}\right) \Gamma\left[m^{\prime}+1\right] \Gamma\left[n^{\prime}+1\right]}
\end{aligned}
$$

where $a=e^{w_{0} / 2}$ and $m^{\prime}, n^{\prime}$ are negative integers.

For states containing one oscillator excitation on the vacuum $\left|0_{R}^{-}\right\rangle\left|0_{R}^{-}\right\rangle$, we have

$$
\begin{aligned}
\sigma_{2}^{+}\left(w_{0}\right) \alpha_{B \dot{B}, n}^{(\mathcal{A})}\left|0_{R}^{-}\right\rangle\left|0_{R}^{-}\right\rangle \\
=2 \sum_{p^{\prime} \leq-1} \frac{i}{\pi} \frac{\Gamma\left[\frac{1}{2}-n\right]}{\Gamma[-n]} \frac{\Gamma\left[-\frac{1}{2}-p^{\prime}\right]}{\Gamma\left[-p^{\prime}\right]} \frac{a^{2\left(n-p^{\prime}\right)-1}}{2 n-2 p^{\prime}-1} \alpha_{B \dot{B}, p^{\prime}+1 / 2}|\chi\rangle \\
\sigma_{2}^{+}\left(w_{0}\right) d_{n}^{+B(\mathcal{A})}\left|0_{R}^{-}\right\rangle\left|0_{R}^{-}\right\rangle \\
=2 \sum_{p^{\prime} \leq-1} \frac{i}{\pi} \frac{\Gamma\left[\frac{1}{2}-n\right]}{\Gamma[1-n]} \frac{\Gamma\left[\frac{1}{2}-p^{\prime}\right]}{\Gamma\left[-p^{\prime}\right]} \frac{a^{2\left(n-p^{\prime}\right)-1}}{2 n-2 p^{\prime}-1} d_{p^{\prime}+1 / 2}^{+B}|\chi\rangle
\end{aligned}
$$


and

$$
\begin{aligned}
& \sigma_{2}^{+}\left(w_{0}\right) d_{n}^{-B(\mathcal{A})}\left|0_{R}^{-}\right\rangle\left|0_{R}^{-}\right\rangle \\
& \quad=2 \sum_{p^{\prime} \leq-1} \frac{i}{\pi} \frac{\Gamma\left[\frac{1}{2}-n\right]}{\Gamma[-n]} \frac{\Gamma\left[-\frac{1}{2}-p^{\prime}\right]}{\Gamma\left[-p^{\prime}\right]} \frac{a^{2\left(n-p^{\prime}\right)-1}}{2 n-2 p^{\prime}-1} d_{p^{\prime}+1 / 2}^{-B}|\chi\rangle
\end{aligned}
$$

where $\alpha_{B \dot{B}, n}^{(\mathcal{A})}$ and $d_{n}^{\beta B(\mathcal{A})}$ are the antisymmetric operators defined in (5.8). We use these antisymmetric operators to build primaries in section 5.2.

We can also start with initial states that have more than one oscillator excitation; i.e., we can compute

$$
\sigma_{2}^{+}\left(w_{0}\right)\left(\prod_{i} O_{i,-n_{i}}\right)\left|0_{R}^{-}\right\rangle\left|0_{R}^{-}\right\rangle
$$

The general method to compute the final state in this situation was given in [22]. We first do all possible contractions in $\prod_{i} O_{i,-n_{i}}$ using the following rules

$$
\begin{aligned}
& C\left[\alpha_{A \dot{A}, m}^{(\mathcal{A})} \alpha_{B \dot{B}, n}^{(\mathcal{A})}\right]=2 \epsilon_{A B} \epsilon_{\dot{A} \dot{B}}(2 i a)^{2 m+2 n} \\
& {\left[\sum_{k=0}^{-n-1}{ }^{m} C_{-m-n-k}{ }^{n} C_{k}(n+k)-\sum_{k=0}^{-m-1} \sum_{q=0}^{-n-1}{ }^{m} C_{k}{ }^{n} C_{q}{ }^{m+k} C_{-(n+q)}(-1)^{m-k+1}(n+q)\right]}
\end{aligned}
$$

and

$$
\begin{aligned}
C\left[d_{m}^{(\mathcal{A})+A} d_{n}^{(\mathcal{A})-B}\right]= & 2 \epsilon^{A B}(2 i a)^{2 m+2 n}\left[\sum_{q=0}^{-n-1}{ }^{m-1} C_{-m-n-q}{ }^{n} C_{q}+2 \sum_{q=0}^{-n-1}{ }^{m-1} C_{-m-n-q-1}{ }^{n} C_{q}\right. \\
& +\sum_{k=0}^{-m} \sum_{q=0}^{-n-1}(-1)^{m-k}{ }^{m-1} C_{k}{ }^{n} C_{q}{ }^{m+k-1} C_{-n-q-1} \\
& \left.+2 \sum_{k=0}^{-m-1} \sum_{q=0}^{-n-1}(-1)^{m-k-1}{ }^{m-1} C_{k}{ }^{n} C_{q}{ }^{m+k} C_{-n-q-1}\right]
\end{aligned}
$$

Then each of oscillators left is moved separately to the final state as indicated in the relations (C.3)-(C.5) discussed above where we had only one initial operator.

\section{Twisting strings with winding numbers $k_{1}$ and $k_{2}$}

The right moving part of the operators $\bar{G}_{\dot{A}, 0}^{\bar{\alpha}(P)}$ is given by $\mathcal{P} \bar{\sigma}^{\bar{\alpha}}$. The effect of twist operator $\bar{\sigma}^{\bar{\alpha}}$ on strings with winding numbers $k_{1}$ and $k_{2}$ was found in [62]. Including the projection operator $\mathcal{P}$, the effect of this operator on the ground state is

$$
\begin{aligned}
& \mathcal{P} \bar{\sigma}_{2}^{-}\left|\overline{0}_{R}^{k_{1}-}\right\rangle\left|\overline{0}_{R}^{k_{2}-}\right\rangle=0 \\
& \mathcal{P} \bar{\sigma}_{2}^{+}\left|\overline{0}_{R}^{k_{1}-}\right\rangle\left|\overline{0}_{R}^{k_{2}-}\right\rangle=C_{k_{1}, k_{2}}^{1 / 2}\left|\overline{0}_{R}^{\left(k_{1}+k_{2}\right)-}\right\rangle
\end{aligned}
$$

where

$$
C_{k_{1}, k_{2}}=\frac{k_{1}+k_{2}}{2 k_{1} k_{2}}
$$


A fermionic mode applied to one of the strings before the twist gives rise to a fermionic mode on the joined string obtained after the twist

$$
\begin{aligned}
& \mathcal{P} \bar{\sigma}_{2}^{+} \bar{d}_{0}^{+A\left(k_{1}\right)}\left|\overline{0}_{R}^{k_{1}-}\right\rangle\left|\overline{0}_{R}^{k_{2}-}\right\rangle=\frac{k_{1}}{k_{1}+k_{2}} C_{k_{1}, k_{2}}^{1 / 2} \bar{d}_{0}^{+A\left(k_{1}+k_{2}\right)}\left|\overline{0}_{R}^{\left(k_{1}+k_{2}\right)-}\right\rangle \\
& \mathcal{P} \bar{\sigma}_{2}^{+} \bar{d}_{0}^{+A\left(k_{2}\right)}\left|\overline{0}_{R}^{k_{1}-}\right\rangle\left|\overline{0}_{R}^{k_{2}-}\right\rangle=\frac{k_{2}}{k_{1}+k_{2}} C_{k_{1}, k_{2}}^{1 / 2} \bar{d}_{0}^{+A\left(k_{1}+k_{2}\right)}\left|\overline{0}_{R}^{\left(k_{1}+k_{2}\right)-}\right\rangle
\end{aligned}
$$

Thus

$$
\mathcal{P} \bar{\sigma}_{2}^{+}\left(\frac{1}{k_{1}} \bar{d}_{0}^{+A\left(k_{1}\right)}-\frac{1}{k_{2}} \bar{d}_{0}^{+A\left(k_{2}\right)}\right)\left|\overline{0}_{R}^{k_{1}-}\right\rangle\left|\overline{0}_{R}^{k_{2}-}\right\rangle=0
$$

We can also consider the action of $\mathcal{P} \bar{\sigma}^{-}$, but in this case we see that the final state on the $k_{1}+k_{2}$ wound string must have charge $\bar{\alpha}=-1$, which is not possible for a Ramond ground state. Thus we have

$$
\mathcal{P} \bar{\sigma}_{2}^{-}\left(\frac{1}{k_{1}} \bar{d}_{0}^{+A\left(k_{1}\right)}-\frac{1}{k_{2}} \bar{d}_{0}^{+A\left(k_{2}\right)}\right)\left|\overline{0}_{R}^{k_{1}-}\right\rangle\left|\overline{0}_{R}^{k_{2}-}\right\rangle=0
$$

For the case where we have two $\bar{d}_{0}$ operators on the initial strings, each such operator can be moved to the final state string using (D.4) and (D.5). We thus have

$$
\mathcal{P} \bar{\sigma}_{2}^{+}\left(\frac{1}{k_{1}} \bar{d}_{0}^{++\left(k_{1}\right)}-\frac{1}{k_{2}} \bar{d}_{0}^{++\left(k_{2}\right)}\right)\left(\frac{1}{k_{1}} \bar{d}_{0}^{+-\left(k_{1}\right)}-\frac{1}{k_{2}} \bar{d}_{0}^{+-\left(k_{2}\right)}\right)\left|\overline{0}_{R}^{k_{1}-}\right\rangle\left|\overline{0}_{R}^{k_{2}-}\right\rangle=0
$$

In general there is also a contraction term between the two initial state operators. However this contraction term will vanish in our present situation, since both the $\bar{d}_{0}$ operators have positive $\bar{\alpha}$ charge, and so they cannot contract with each other.

Now consider the action of $\mathcal{P} \bar{\sigma}^{-}$on the state with two initial excitations

$$
\begin{aligned}
\mathcal{P} \bar{\sigma}_{2}^{-} & \left(\frac{1}{k_{1}} \bar{d}_{0}^{++\left(k_{1}\right)}-\frac{1}{k_{2}} \bar{d}_{0}^{++\left(k_{2}\right)}\right)\left(\frac{1}{k_{1}} \bar{d}_{0}^{+-\left(k_{1}\right)}-\frac{1}{k_{2}} \bar{d}_{0}^{+-\left(k_{2}\right)}\right)\left|\overline{0}_{R}^{k_{1}-}\right\rangle\left|\overline{0}_{R}^{k_{2}-}\right\rangle \\
& =\left[\bar{J}_{0}^{-}, \mathcal{P} \bar{\sigma}_{2}^{+}\right]\left(\frac{1}{k_{1}} \bar{d}_{0}^{++\left(k_{1}\right)}-\frac{1}{k_{2}} \bar{d}_{0}^{++\left(k_{2}\right)}\right)\left(\frac{1}{k_{1}} \bar{d}_{0}^{+-\left(k_{1}\right)}-\frac{1}{k_{2}} \bar{d}_{0}^{+-\left(k_{2}\right)}\right)\left|\overline{0}_{R}^{k_{1}-}\right\rangle\left|\overline{0}_{R}^{k_{2}-}\right\rangle \\
& =-\mathcal{P} \bar{\sigma}_{2}^{+} \bar{J}_{0}^{-}\left(\frac{1}{k_{1}} \bar{d}_{0}^{++\left(k_{1}\right)}-\frac{1}{k_{2}} \bar{d}_{0}^{++\left(k_{2}\right)}\right)\left(\frac{1}{k_{1}} \bar{d}_{0}^{+-\left(k_{1}\right)}-\frac{1}{k_{2}} \bar{d}_{0}^{+-\left(k_{2}\right)}\right)\left|\overline{0}_{R}^{k_{1}-}\right\rangle\left|\overline{0}_{R}^{k_{2}-}\right\rangle \\
& =-\mathcal{P} \bar{\sigma}_{2}^{+}\left(\frac{1}{k_{1}} \bar{d}_{0}^{-+\left(k_{1}\right)}-\frac{1}{k_{2}} \bar{d}_{0}^{-+\left(k_{2}\right)}\right)\left(\frac{1}{k_{1}} \bar{d}_{0}^{+-\left(k_{1}\right)}-\frac{1}{k_{2}} \bar{d}_{0}^{+-\left(k_{2}\right)}\right)\left|\overline{0}_{R}^{k_{1}-}\right\rangle\left|\overline{0}_{R}^{k_{2}-}\right\rangle \\
& =-\mathcal{P} \bar{\sigma}_{2}^{+}\left(\frac{1}{k_{1}}+\frac{1}{k_{2}}\right)\left|\overline{0}_{R}^{k_{1}-}\right\rangle\left|\overline{0}_{R}^{k_{2}-}\right\rangle \\
& \left.=-\frac{k_{1}+k_{2}}{k_{1} k_{2}} C_{k_{1}, k_{2}}^{1 / 2} \overline{0}_{R}^{\left(k_{1}+k_{2}\right)-}\right\rangle
\end{aligned}
$$

where to get the third line we used (D.8) and to get the fifth line we used the commutator (A.2).

In summary, we find that the operators $\bar{G}_{\dot{A}, 0}^{\bar{\alpha}(P)}$ annihilate the right moving states which contain one operator of the type (7.12); this is shown in (D.6) and (D.7). But the operators $\bar{G}_{\dot{A}, 0}^{\bar{\alpha}(P)}$ do not annihilate the right moving sector containing zero or two operators of the type (7.12); this is shown by (D.2) and (D.9). 
Open Access. This article is distributed under the terms of the Creative Commons Attribution License (CC-BY 4.0), which permits any use, distribution and reproduction in any medium, provided the original author(s) and source are credited.

\section{References}

[1] A. Strominger and C. Vafa, Microscopic origin of the Bekenstein-Hawking entropy, Phys. Lett. B 379 (1996) 99 [hep-th/9601029] [INSPIRE].

[2] C.G. Callan and J.M. Maldacena, D-brane approach to black hole quantum mechanics, Nucl. Phys. B 472 (1996) 591 [hep-th/9602043] [INSPIRE].

[3] S.R. Das and S.D. Mathur, Comparing decay rates for black holes and D-branes, Nucl. Phys. B 478 (1996) 561 [hep-th/9606185] [INSPIRE].

[4] J.M. Maldacena and A. Strominger, Black hole grey body factors and D-brane spectroscopy, Phys. Rev. D 55 (1997) 861 [hep-th/9609026] [INSPIRE].

[5] C. Vafa, Instantons on D-branes, Nucl. Phys. B 463 (1996) 435 [hep-th/9512078] [INSPIRE].

[6] R. Dijkgraaf, Instanton strings and hyperKähler geometry, Nucl. Phys. B 543 (1999) 545 [hep-th/9810210] [INSPIRE].

[7] N. Seiberg and E. Witten, The D1/D5 system and singular CFT, JHEP 04 (1999) 017 [hep-th/9903224] [INSPIRE].

[8] F. Larsen and E.J. Martinec, U(1) charges and moduli in the D1-D5 system, JHEP 06 (1999) 019 [hep-th/9905064] [INSPIRE].

[9] G.E. Arutyunov and S.A. Frolov, Virasoro amplitude from the $S^{N} R^{24}$ orbifold sigma model, Theor. Math. Phys. 114 (1998) 43 [hep-th/9708129] [inSPIRE].

[10] G.E. Arutyunov and S.A. Frolov, Four graviton scattering amplitude from $S^{N} R^{8}$ supersymmetric orbifold sigma model, Nucl. Phys. B 524 (1998) 159 [hep-th/9712061] [INSPIRE].

[11] A. Jevicki, M. Mihailescu and S. Ramgoolam, Gravity from $C F T$ on $S^{N}(X)$ : Symmetries and interactions, Nucl. Phys. B 577 (2000) 47 [hep-th/9907144] [InSPIRE].

[12] J.R. David, G. Mandal and S.R. Wadia, Microscopic formulation of black holes in string theory, Phys. Rept. 369 (2002) 549 [hep-th/0203048] [INSPIRE].

[13] J.M. Maldacena, G.W. Moore and A. Strominger, Counting BPS black holes in toroidal Type II string theory, hep-th/9903163 [INSPIRE].

[14] O. Lunin and S.D. Mathur, AdS/CFT duality and the black hole information paradox, Nucl. Phys. B 623 (2002) 342 [hep-th/0109154] [INSPIRE].

[15] S.D. Mathur, The Fuzzball proposal for black holes: An Elementary review, Fortsch. Phys. 53 (2005) 793 [hep-th/0502050] [INSPIRE].

[16] I. Kanitscheider, K. Skenderis and M. Taylor, Fuzzballs with internal excitations, JHEP 06 (2007) 056 [arXiv:0704.0690] [INSPIRE].

[17] I. Bena and N.P. Warner, Black holes, black rings and their microstates, in Lecture Notes in Physics 755, Springer (2008), pp. 1-92 [hep-th/0701216] [INSPIRE]. 
[18] B.D. Chowdhury and A. Virmani, Modave Lectures on Fuzzballs and Emission from the D1-D5 System, in proceedings of the 5th Modave Summer School in Mathematical Physics, Modave, Belgium, 17-21 August 2009, arXiv:1001.1444 [INSPIRE].

[19] E. Gava and K.S. Narain, Proving the PP-wave/CFT 2 duality, JHEP 12 (2002) 023 [hep-th/0208081] [INSPIRE].

[20] B. Guo and S.D. Mathur, Lifting of level-1 states in the D1D5 CFT, JHEP 03 (2020) 028 [arXiv: 1912.05567] [INSPIRE].

[21] S.G. Avery, B.D. Chowdhury and S.D. Mathur, Deforming the D1D5 CFT away from the orbifold point, JHEP 06 (2010) 031 [arXiv:1002.3132] [INSPIRE].

[22] S.G. Avery, B.D. Chowdhury and S.D. Mathur, Excitations in the deformed D1D5 CFT, JHEP 06 (2010) 032 [arXiv: 1003.2746] [inSPIRE].

[23] A. Pakman, L. Rastelli and S.S. Razamat, Diagrams for Symmetric Product Orbifolds, JHEP 10 (2009) 034 [arXiv:0905.3448] [INSPIRE].

[24] A. Pakman, L. Rastelli and S.S. Razamat, Extremal Correlators and Hurwitz Numbers in Symmetric Product Orbifolds, Phys. Rev. D 80 (2009) 086009 [arXiv:0905.3451] [InSPIRE].

[25] A. Pakman, L. Rastelli and S.S. Razamat, A Spin Chain for the Symmetric Product CFT $_{2}$, JHEP 05 (2010) 099 [arXiv:0912.0959] [INSPIRE].

[26] B.A. Burrington, A.W. Peet and I.G. Zadeh, Operator mixing for string states in the D1-D5 CFT near the orbifold point, Phys. Rev. D 87 (2013) 106001 [arXiv:1211.6699] [INSPIRE].

[27] B.A. Burrington, A.W. Peet and I.G. Zadeh, Twist-nontwist correlators in $M^{N} / S_{N}$ orbifold CFTs, Phys. Rev. D 87 (2013) 106008 [arXiv:1211.6689] [inSPIRE].

[28] B.A. Burrington, S.D. Mathur, A.W. Peet and I.G. Zadeh, Analyzing the squeezed state generated by a twist deformation, Phys. Rev. D 91 (2015) 124072 [arXiv:1410.5790] [INSPIRE].

[29] B.A. Burrington, I.T. Jardine and A.W. Peet, Operator mixing in deformed D1D5 CFT and the OPE on the cover, JHEP 06 (2017) 149 [arXiv:1703.04744] [INSPIRE].

[30] Z. Carson, S. Hampton and S.D. Mathur, Full action of two deformation operators in the D1D5 CFT, JHEP 11 (2017) 096 [arXiv:1612.03886] [INSPIRE].

[31] Z. Carson, S. Hampton and S.D. Mathur, One-Loop Transition Amplitudes in the D1D5 CFT, JHEP 01 (2017) 006 [arXiv:1606.06212] [INSPIRE].

[32] Z. Carson, S. Hampton and S.D. Mathur, Second order effect of twist deformations in the D1D5 CFT, JHEP 04 (2016) 115 [arXiv:1511.04046] [INSPIRE].

[33] Z. Carson, S. Hampton, S.D. Mathur and D. Turton, Effect of the deformation operator in the D1D5 CFT, JHEP 01 (2015) 071 [arXiv:1410.4543] [INSPIRE].

[34] Z. Carson, S.D. Mathur and D. Turton, Bogoliubov coefficients for the twist operator in the D1D5 CFT, Nucl. Phys. B 889 (2014) 443 [arXiv:1406.6977] [InSPIRE].

[35] Z. Carson, S. Hampton, S.D. Mathur and D. Turton, Effect of the twist operator in the D1D5 CFT, JHEP 08 (2014) 064 [arXiv:1405.0259] [INSPIRE].

[36] L.P. Kadanoff, Multicritical behavior at the Kosterlitz-Thouless critical point, Ann. Phys. 120 (1979) 39. 
[37] R. Dijkgraaf, E.P. Verlinde and H.L. Verlinde, On Moduli Spaces Of Conformal Field Theories With $c \geq 1$, in Perspectives in String Theory, World Scientific, Copenhagen Denmark (1987), pp. 117-137 [INSPIRE].

[38] J.L. Cardy, Continuously Varying Exponents and the Value of the Central Charge, J. Phys. A 20 (1987) L891 [INSPIRE].

[39] D. Kutasov, Geometry on the Space of Conformal Field Theories and Contact Terms, Phys. Lett. B 220 (1989) 153 [INSPIRE].

[40] H. Eberle, Twistfield perturbations of vertex operators in the $\mathbb{Z}_{2}$-orbifold model, Ph.D. Thesis, University of Bonn, Bonn Germany (2006) [JHEP 06 (2002) 022] [hep-th/0103059] [INSPIRE].

[41] M.R. Gaberdiel, A. Konechny and C. Schmidt-Colinet, Conformal perturbation theory beyond the leading order, J. Phys. A 42 (2009) 105402 [arXiv:0811.3149] [InSPIRE].

[42] D. Berenstein and A. Miller, Conformal perturbation theory, dimensional regularization, and AdS/CFT correspondence, Phys. Rev. D 90 (2014) 086011 [arXiv:1406.4142] [InSPIRE].

[43] D. Berenstein and A. Miller, Logarithmic enhancements in conformal perturbation theory and their real time interpretation, Int. J. Mod. Phys. A 35 (2020) 2050184 [arXiv:1607.01922] [INSPIRE].

[44] M.R. Gaberdiel, C. Peng and I.G. Zadeh, Higgsing the stringy higher spin symmetry, JHEP 10 (2015) 101 [arXiv: 1506. 02045] [INSPIRE].

[45] S. Hampton, S.D. Mathur and I.G. Zadeh, Lifting of D1-D5-P states, JHEP 01 (2019) 075 [arXiv: 1804.10097] [INSPIRE].

[46] C.A. Keller and I.G. Zadeh, Lifting $\frac{1}{4}$-BPS States on K3 and Mathieu Moonshine, Commun. Math. Phys. 377 (2020) 225 [arXiv:1905.00035] [InSPIRE].

[47] C.A. Keller and I.G. Zadeh, Conformal Perturbation Theory for Twisted Fields, J. Phys. A 53 (2020) 095401 [arXiv: 1907.08207] [INSPIRE].

[48] A. Sevrin, W. Troost and A. Van Proeyen, Superconformal Algebras in Two-Dimensions with $N=4$, Phys. Lett. B 208 (1988) 447 [InSPIRE].

[49] B. Guo and S.D. Mathur, Lifting of states in 2-dimensional $N=4$ supersymmetric CFTs, JHEP 10 (2019) 155 [arXiv: 1905.11923] [INSPIRE].

[50] N. Benjamin, A Refined Count of BPS States in the D1/D5 System, JHEP 06 (2017) 028 [arXiv: 1610.07607] [INSPIRE].

[51] N. Benjamin and S.M. Harrison, Symmetries of the refined D1/D5 BPS spectrum, JHEP 11 (2017) 091 [arXiv: 1708.02244] [INSPIRE].

[52] I. Bena, S. Giusto, R. Russo, M. Shigemori and N.P. Warner, Habemus Superstratum! A constructive proof of the existence of superstrata, JHEP 05 (2015) 110 [arXiv:1503.01463] [INSPIRE].

[53] I. Bena, E. Martinec, D. Turton and N.P. Warner, Momentum Fractionation on Superstrata, JHEP 05 (2016) 064 [arXiv: 1601.05805] [InSPIRE].

[54] I. Bena et al., Smooth horizonless geometries deep inside the black-hole regime, Phys. Rev. Lett. 117 (2016) 201601 [arXiv: 1607.03908] [INSPIRE].

[55] I. Bena et al., Asymptotically-flat supergravity solutions deep inside the black-hole regime, JHEP 02 (2018) 014 [arXiv:1711.10474] [INSPIRE]. 
[56] N. Čeplak, R. Russo and M. Shigemori, Supercharging Superstrata, JHEP 03 (2019) 095 [arXiv: 1812.08761] [INSPIRE].

[57] P. Heidmann and N.P. Warner, Superstratum Symbiosis, JHEP 09 (2019) 059 [arXiv: 1903.07631] [INSPIRE].

[58] T. Eguchi and A. Taormina, Unitary Representations of $N=4$ Superconformal Algebra, Phys. Lett. B 196 (1987) 75 [InSPIRE].

[59] T. Eguchi and A. Taormina, Character Formulas for the $N=4$ Superconformal Algebra, Phys. Lett. B 200 (1988) 315 [INSPIRE].

[60] J.L. Petersen and A. Taormina, Characters of the $N=4$ Superconformal Algebra With Two Central Extensions, Nucl. Phys. B 331 (1990) 556 [InSPIRE].

[61] J.L. Petersen and A. Taormina, Characters of the $N=4$ Superconformal Algebra With Two Central Extensions: 2. Massless Representations, Nucl. Phys. B 333 (1990) 833 [inSPIRE].

[62] Z. Carson, S. Hampton, S.D. Mathur and D. Turton, Effect of the deformation operator in the D1D5 CFT, JHEP 01 (2015) 071 [arXiv: 1410.4543] [INSPIRE]. 